\title{
Establishing a Role of the Semantic Control Network in Social Cognitive Processing: A Meta-analysis of Functional Neuroimaging Studies
}

Diveica, Veronica; Koldewyn, Kami; Binney, Richard

\section{Neuroimage}

DOI:

https://doi.org/10.1016/j.neuroimage.2021.118702

Published: $15 / 12 / 2021$

Publisher's PDF, also known as Version of record

Cyswllt i'r cyhoeddiad / Link to publication

Dyfyniad o'r fersiwn a gyhoeddwyd / Citation for published version (APA):

Diveica, V., Koldewyn, K., \& Binney, R. (2021). Establishing a Role of the Semantic Control Network in Social Cognitive Processing: A Meta-analysis of Functional Neuroimaging Studies. Neuroimage, 245, [118702]. https://doi.org/10.1016/j.neuroimage.2021.118702

\footnotetext{
Hawliau Cyffredinol / General rights

Copyright and moral rights for the publications made accessible in the public portal are retained by the authors and/or other copyright owners and it is a condition of accessing publications that users recognise and abide by the legal requirements associated with these rights.

- Users may download and print one copy of any publication from the public portal for the purpose of private study or research.

- You may not further distribute the material or use it for any profit-making activity or commercial gain

- You may freely distribute the URL identifying the publication in the public portal?
}

Take down policy

If you believe that this document breaches copyright please contact us providing details, and we will remove access to the work immediately and investigate your claim. 


\title{
Establishing a role of the semantic control network in social cognitive processing: A meta-analysis of functional neuroimaging studies
}

\author{
Veronica Diveica, Kami Koldewyn, Richard J. Binney* \\ School of Human and Behavioural Sciences, Bangor University, Bangor, Gwynedd, Wales, LL57 2AS, United Kingdom
}

\section{A R T I C L E I N F O}

\section{Keywords:}

Cognitive control

Empathy

Theory of mind

Moral reasoning

Trait inference

Meta-analysis

\begin{abstract}
A B S T R A C T
The contribution and neural basis of cognitive control is under-specified in many prominent models of sociocognitive processing. Important outstanding questions include whether there are multiple, distinguishable systems underpinning control and whether control is ubiquitously or selectively engaged across different social behaviours and task demands. Recently, it has been proposed that the regulation of social behaviours could rely on brain regions specialised in the controlled retrieval of semantic information, namely the anterior inferior frontal gyrus (IFG) and posterior middle temporal gyrus. Accordingly, we investigated for the first time whether the neural activation commonly found in social functional neuroimaging studies extends to these 'semantic control' regions. We conducted five coordinate-based meta-analyses to combine results of $499 \mathrm{fMRI} / \mathrm{PET}$ experiments and identified the brain regions consistently involved in semantic control, as well as four social abilities: theory of mind, trait inference, empathy and moral reasoning. This allowed an unprecedented parallel review of the neural networks associated with each of these cognitive domains. The results confirmed that the anterior left IFG region involved in semantic control is reliably engaged in all four social domains. This supports the hypothesis that social cognition is partly regulated by the neurocognitive system underpinning semantic control.
\end{abstract}

\section{Introduction}

The ability to comprehend and respond appropriately to the behaviour of others is essential for humans to survive and thrive. A major challenge for the cognitive sciences, therefore, is to characterise how we understand others and coordinate our behaviour to achieve mutually beneficial outcomes, and what can cause this ability to break down (Frith, 2007). There is an indubitable requirement for systems that control, or regulate, the cognitive processes underpinning social interactions. This is because social interactions are intricate and fraught with the potential for misunderstandings and faux pas; first, the everyday social signals to which we are exposed are typically complex, often ambiguous and sometimes conflicting. This is compounded by the fact that the meaning of a given gesture, expression or utterance can vary across contexts (Barrett et al., 2011; Rodd, 2020). Moreover, once we have settled upon an interpretation of these signals, we are then faced with the additional challenge of selecting an appropriate response, and inhibiting others which might, for example, be utilitarian but socially insensitive or even damaging. In order to undergo social interactions that are coherent, effective and context-appropriate, we must carefully regulate both our comprehension of, and response to, the intentions and actions of others (Binney and Ramsey, 2020; Fujita et al., 2014; Gilbert and Burgess, 2008; Ramsey and Ward, 2020).

\footnotetext{
* Corresponding author.

E-mail address: R.Binney@Bangor.ac.uk (R.J. Binney).
}

Despite there being a wealth of literature describing executive functions involved in general cognition (Assem et al., 2020; Diamond, 2013; Duncan, 2013, 2010; Fedorenko et al., 2013; Petersen and Posner, 2012), prominent models of socio-cognitive processing are underspecified in terms of the contribution and neural basis of cognitive control mechanisms (e.g., Adolphs, 2009, 2010; Frith and Frith, 2012; Lieberman, 2007). For example, Adolphs (2009, 2010) only very briefly refers to cognitive processes involved in 'social regulation' and largely within the limited context of emotional regulation. Likewise, Frith and Frith (2012) refer to a "supervisory system" which has the characteristic features of executive control, but its functional and anatomical descriptions lack detail important for generating testable hypotheses. However, research into specific social phenomena, such as prejudice (Amodio, 2014; Amodio and Cikara, 2021) and automatic imitation (Cross et al., 2013; Darda and Ramsey, 2019) has recently begun to give the matter of cognitive control greater attention. Of particular interest has been the contribution of the domain-general multipledemand network (MDN), a set of brain areas engaged by cognitivelychallenging tasks irrespective of the cognitive domain (Assem et al., 2020; Duncan, 2010; Fedorenko et al., 2013; Hugdahl et al., 2015). MDN activity increases with many kinds of general task demand, including working memory load and task switching, and it has been suggested that this reflects the implementation of top-down attentional control and the optimal allocation of cognitive resources to meet immediate goals (Duncan, 2013, 2010). The MDN is comprised of parts of the precentral gyrus, the middle frontal gyrus (MFG), the intraparietal sulcus (IPS), in- 
sular cortex, the pre-supplementary motor area (pre-SMA) and the adjacent cingulate cortex (Assem et al., 2020; Fedorenko et al., 2013), some of which have been implicated in controlled social processing such as, for example, working memory for social content (Meyer et al., 2012), social conflict resolution (Zaki et al., 2010), inhibition of automatic imitation (Darda and Ramsey, 2019) and mental state inference or theory of mind (ToM) (e.g. Rothmayr et al., 2011; Samson et al., 2005; Van der Meer et al., 2011). However, there are at least three key unresolved questions regarding the role of cognitive control in social cognition. First, it remains to be seen whether there could be multiple, distinguishable types of, and neural systems for, control. Second, it is unclear whether distinguishable control systems are necessary for all or only certain social abilities and, third, whether this engagement depends on specific task demands. Shedding light on these issues has the potential to generate important new hypotheses regarding social behaviour both in the context of health and injury/disease.

It has recently been proposed that a relatively specialised form of cognitive control, termed semantic control, could be particularly important for social cognitive processing (Binney and Ramsey, 2020). This proposal argued that a semantic control system is required during social cognitive tasks to modulate the retrieval and selection of conceptuallevel knowledge so that it is relevant to the situational context or the task at hand (Chiou et al., 2018; Jefferies, 2013; Lambon Ralph et al., 2017). The reasons why semantic control should be critical for social cognition are uncomplicated; we retain a vast amount of socially-relevant knowledge including knowledge about familiar people (Greven et al., 2016; Hassabis et al., 2014), about the structure of and relationship between social categories and their associated stereotypes (Freeman and Johnson, 2016; Quinn and Rosenthal, 2012), and an understanding of abstract social concepts, norms and scripts (Frith and Frith, 2003; Van Overwalle, 2009). But only a limited portion of this information is relevant in a given social instance and it would be computationally inefficient to automatically retrieve it all. For example, there is no need to retrieve information about someone's personality traits, or personal interests and hobbies, if the only task is to pick them out from within a crowd. Moreover, the types and the scope of information we need to retrieve to understand and respond appropriately to certain social signals change according to the context, and irrelevant information could potentially interfere. Therefore, semantic control should be essential for limiting potential social errors.

There is a growing body of convergent patient, neuroimaging and neuromodulation evidence that semantic memory retrieval engages the semantic control network (SCN) which comprises the anterior IFG and the posterior middle temporal gyrus (pMTG) (Badre et al., 2005; Jackson, 2021; Noonan et al., 2010; Whitney et al., 2012). While the domain-general MDN is also engaged by semantic tasks, and particularly those with high control demands (Jackson, 2021; Thompson et al., 2018), there is evidence to suggest that both the anatomy of the SCN and MDN and their functional contributions to controlled semantic processing are distinct (Gao et al., 2020; Humphreys and Lambon Ralph, 2017; Wang et al., 2018; Whitney et al., 2012). In particular, fMRI studies reveal that the mid- to posterior IFG (pars triangularis and pars opercularis), nodes of the MDN, have been shown to increase activity in response to increased 'semantic selection' demands, a process that is engaged when automatic retrieval of semantic knowledge results in competition between multiple representations which must be resolved (for example, hearing the word bank might elicit retrieval of the concept of a riverside and a financial institution)(Badre et al., 2005; Nagel et al., 2008; Thompson-Schill et al., 1997). However, this mid- to posterior IFG region is also engaged by other non-semantic forms of response competition (Badre and Wagner, 2007; Dobbins and Wagner, 2005) and tests of inhibitory function such as the Stroop task (Huang et al., 2020; January et al., 2009; Nee et al., 2007). In contrast, activation of the anterior IFG (pars orbitalis) appears to be more selective to semantic control demands and driven specifically by an increased need for 'controlled semantic retrieval', a mechanism that is engaged when automatic semantic retrieval fails to activate semantic information necessary for the task at hand, and a further goal-directed semantic search needs to be initiated (Gold et al., 2006; Krieger-Redwood et al., 2015; Wagner et al., 2001).

To date, there have been but a few neuroimaging investigations that have directly questioned the involvement of the SCN in social cognitive processing. Two recent fMRI studies compared activation during semantic judgements made on social and non-social stimuli and found that the IFG and pMTG were engaged by both stimulus types (Binney et al., 2016b; Rice et al., 2018). Further, Satpute et al. (2014) found that controlled retrieval, but not selection of social conceptual information engages the anterior IFG. However, we are not aware of any prior studies that attempt to examine the engagement of the SCN specifically during tasks that are commonly viewed as social in nature (e.g., ToM tasks). As a starting point, rather than conducting a novel individual experiment, the present study adopted a meta-analytic approach to extract reliable trends from large numbers of studies. Meta-analyses of functional neuroimaging data overcome the limitations of individual studies (Cumming, 2014; Eickhoff et al., 2012), which are frequently statistically underpowered (Button et al., 2013) and vulnerable to effects of idiosyncratic design and analytic choices (Botvinik-Nezer et al., 2020; Carp, 2012) so that it becomes difficult to distinguish between replicable and spurious findings and to generalize the results. Our principal aim was to determine whether the distributed neural activation commonly associated with functional neuroimaging studies of social cognition extends to the neural networks underpinning semantic control (i.e., SCN and MDN). In order to localise the brain network sensitive to semantic control demands (i.e., semantic retrieval and/or selection), and then compare and contrast it to networks implicated in social cognition, we performed an update of Noonan et al. (2013) meta-analysis of semantic control (also see Jackson, 2021 for another updated meta-analysis of semantic control which additionally investigated the effect of input modality).

We took the approach of investigating multiple sub-domains of social cognition in parallel because this should allow an assessment of the extent to which inferences are generalisable, rather than specific to certain types of social tasks and/or abilities. We chose to focus on four particular areas of research that target abilities frequently identified as key facets of the human social repertoire - ToM, empathy, trait inference, and moral reasoning (Lieberman, 2007; Van Overwalle, 2009) - and, for each, we conducted separate meta-analyses of the available functional imaging data to determine the brain regions consistently implicated. In the case of trait inference, this was the first neuroimaging meta-analysis to include studies that used stimuli other than faces (see Section 2, and also Bzdok et al., 2011, and Mende-Siedlecki et al., 2013, for contrasting approaches). In the other three cases, we performed updates of prior meta-analyses (Eres et al., 2018; Molenberghs et al., 2016; Timmers et al., 2018).

Further, we conducted an exploratory conjunction analysis aimed at identifying brain areas reliably implicated in all four social subdomains and, thus, a core network for social cognitive processing (also see Bzdok et al., 2012; Schurz et al., 2020; Van Overwalle, 2009). We hypothesised that this core network would include parts of the MDN and the SCN. It is of note that, across all four social sub-domains, we took a different approach to study inclusion and exclusion criteria than that taken by some prior meta-analyses of general social cognition (e.g., Van Overwalle, 2009). In particular, we excluded studies investigating processes associated primarily with the self because social cognition is, although perhaps only in the strictest sense, about understanding other people. We also excluded studies in which tasks could be completed based on relatively simple perceptual processing and without a need for deeper cognitive and inferential processes (e.g., emotion discrimination tasks, automatic imitation). This was done in an attempt to constrain our inferences to be about the neurobiology underpinning cognitive rather than primarily perceptual social processes (for further detail on this distinction, see Adolphs, 2010, and Binney and Ramsey, 2020). 
Finally, as a secondary aim, the present study used the metaanalytic approach to assess whether there are differences in the neural networks engaged by implicit and explicit social processing (also see Dricu and Frühholz, 2016; Eres et al., 2018; Fan et al., 2011; Molenberghs et al., 2016; Timmers et al., 2018). This was aimed at addressing a pervasive distinction in the social neuroscientific literature between automatic and controlled processes (Adolphs, 2010; Happé et al., 2017; Lieberman, 2007), and followed an assumption that implicit paradigms engage only automatic processes, whereas controlled processes are recruited during explicit paradigms (Sherman et al., 2014). Automatic processes are described as unintentional, effortless, and fast, whereas controlled processes are deliberate, effortful, and thus slower (Lieberman, 2007; Shiffrin and Schneider, 1977). Some authors have argued that automatic and controlled social processes are mutually exclusive of one another and draw upon distinct cortical networks, with the former engaging lateral temporal cortex, the amygdala, ventromedial frontal cortex and the anterior cingulate, and the latter engaging lateral and medial prefrontal and parietal cortex (Forbes and Grafman, 2013; Lieberman, 2007). However, these dual-process models have been criticised for over-simplifying both the distinction and the relationship between automatic and controlled processes (Amodio, 2019; Cunningham and Zelazo, 2007; Ferguson et al., 2014; Fidler and Hütter, 2014; Fujita et al., 2014; Melnikoff and Bargh, 2018). An alternative proposal, that we describe above, makes a different distinction - one between representation and control. This neurocognitive model proposes that social processing relies on a single-route architecture wherein the degree to which cognitive processing has certain attributes (e.g., speed or effort) does not reflect one system versus another. Instead, it is proposed that it reflects the degree to which the control system needs to exert influence, upon otherwise automatic activation within the representational system, in order to meet the demands of a task in an appropriate and efficient manner (Binney and Ramsey, 2020; Jefferies, 2013). If the dual route model is correct, explicit but not implicit social paradigms should differentially engage brain regions associated with cognitive control demands, including the SCN and MDN. If the single-route model is correct, then there should be no qualitative difference in terms of the network of regions activated by implicit paradigms (ergo automatic processing) and explicit paradigms (ergo controlled processing), although there may be differences in the magnitude of regional activation.

To summarise, the aims of the present study were as follows: 1) explore the involvement of domain-general control systems in social cognition; more specifically, determine whether social cognitive processing reliably engages brain areas implicated in the controlled retrieval and selection of conceptual knowledge; and 2) examine the evidence for dual-route and single-route models of controlled social cognition.

\section{Methods}

Preregistration and Open Science statement. Following open science initiatives (Munafò et al., 2017), the current study was pre-registered via the Open Science Framework (OSF). We adhered to our pre-registered protocols (available at: osf.io/dscwv) with a few minor exceptions (see Section S1 of Supplementary Information (SI) 1 for details). All the raw datasets are openly-available on the OSF project page (available at: osf.io/fktb8/) and are accompanied by a range of study characteristics including details that are not the focus of the present study but may be of interest in future research (please see Section S1 of SI1 for a detailed description). Moreover, the input data and output files of all analyses can be accessed via the OSF page.

In accordance with our pre-registered aims, we performed a comprehensive review of published functional neuroimaging studies investigating four social abilities - Theory of mind (ToM), trait inference, empathy and moral reasoning - and independent coordinate-based metaanalyses aimed at characterising the brain-wide neural networks underpinning each. In the case of three of these domains (ToM, empathy and moral reasoning), we updated earlier meta-analyses (Eres et al.,
2018; Molenberghs et al., 2016; Timmers et al., 2018), capitalizing on additional data, and also implementing recommendations for best practice that became available in a year subsequent to these prior studies (Müller et al., 2018). In the case of trait inference, as far as we are aware, this was the first neuroimaging meta-analysis to include studies that explored potential sources of information beyond face stimuli (for contrasting approaches see Bzdok et al., 2011; Mende-Siedlecki et al., 2013). To localise the brain areas underpinning semantic retrieval and selection, we also updated a meta-analysis of functional imaging studies of semantic control by Noonan et al. (2013). This involved the inclusion of additional data, and improvements in meta-analytic tools which corrected previous implementation errors that led to the use of liberal statistical thresholds (Eickhoff et al., 2017).

To directly address our first aim, we assessed the degree of overlap between the neural networks supporting semantic control and those involved in social information processing via a set of formal conjunctions and contrasts analyses. To address our second aim, where possible, we contrasted brain-wide activation associated with explicit versus implicit social cognitive paradigms. Tasks that drew the participant's attention to the behaviour/cognitive process of interest were categorised as explicit, while tasks that used non-specific instructions (e.g., they involved passive observation of stimuli) or employed orthogonal tasks (e.g., age judgement) were categorised as implicit. Finally, where sufficient relevant information was available, we explored the influence of task difficulty on patterns of brain activation.

All of the meta-analyses reported below were conducted following best-practice guidelines recommended by Müller et al. (2018). This, as well as several refinements to inclusion/exclusion criteria, contributed to methodological differences between the present meta-analyses and those prior meta-analyses upon which the 'updates' were based. A summary of similarities and differences is provided in Table S1 (SI1) and further details are given in the sections below.

\subsection{Literature selection and inclusion criteria}

\subsubsection{General approach and criteria}

Where possible, relevant functional neuroimaging studies were initially identified based on their inclusion in a recent prior neuroimaging meta-analysis. These lists were supplemented via a search on the Web of Science (WoS) online database (www.webofknowledge.com) for original reports published in the years subsequent, and by searching through reference lists of said articles. Each WoS search used the terms ['fMRI' or 'PET'], as well as terms uniquely chosen for a given cognitive domain (see Table 1).

A general set of inclusion criteria applied to all our analyses were as follows:

(1) Only studies that employed task-based fMRI or PET to obtain original data were included. Studies employing other techniques (e.g., EEG/MEG), meta-analyses and review articles were excluded.

(2) Studies were only included if they reported whole-brain activation coordinates that were localised in one of two standardised spaces - Talairach (TAL) or Montreal Neurological Institute (MNI) - or these coordinates were made available on request (see Section S1 of SI1). Coordinates reported in TAL space were converted into MNI space using the Lancaster transform (tal2icbm transform (Lancaster et al., 2007) embedded within the GingerALE software version 3.0.2; http://brainmap.org/ale). Studies exclusively reporting results from region-of-interest or small volume correction analyses were excluded because these types of analysis violate a key assumption of coordinate-based meta-analyses (Eickhoff et al., 2012; Müller et al., 2018).

(3) Studies were only included if they reported activation coordinates that resulted from univariate contrasts clearly aimed at identifying the process of interest (e.g., ToM). We included contrasts between an experimental task and either a comparable active control task or a 
Table 1

Terms used to search the Web of Science database for relevant articles.

\begin{tabular}{ll}
\hline Cognitive domain & Search terms \\
\hline Semantic control & 'semantic', 'comprehension', 'conceptual knowledge', 'selection', 'retrieval', 'inhibition', 'control', 'elaboration', 'fluency', 'ambiguity', 'metaphor', 'idiom' \\
Theory of Mind & 'theory of mind', 'ToM', 'mentalising', 'mentalizing' \\
Trait inference & 'social judgement', 'social evaluation', 'social attribution', 'trait inference', 'impression formation' \\
Empathy & 'empathy', plus 'empath"' - corresponding variations (e.g. 'empathic') \\
Moral cognition & 'morality', 'moral', 'moral decision making', 'moral emotion', 'harm', 'guilt' \\
\hline
\end{tabular}

N.b., For all five cognitive domains, the search followed the following format: [fMRI OR PET] AND [term1 OR term2 OR ... OR termX].

low-level baseline such as rest or passive fixation. Contrasts against low-level baselines were included in the primary analyses because they can reveal activity associated with domain-general cognitive processes that is subtracted out by contrasts between active conditions. This could include semantic processes that are common to both social and non-social tasks. However, contrasts against lowlevel baselines also yield activity associated with differences in perceptual stimulation and attentional demand. To address this caveat, we repeated the analyses whilst excluding this subset of contrasts. The results can be found on the project's OSF page (available at: osf.io/fktb8/). We excluded contrasts that make comparisons between components of the process of interest (e.g., affective vs. cognitive ToM; utilitarian vs. deontological moral judgements) because we were interested in the common, core processes that would be subtracted out by these contrasts (but see the following paragraph).

(4) Multiple contrasts from a single group of participants (e.g., separate contrasts against one of two different baseline conditions) were included in a single meta-analysis as long as they independently met all other inclusion criteria for the primary analyses. This allowed maximum use of all available data and enabled us to evaluate the effect of using different types of baseline, for example (see above). However, it is important to adjust for this (Müller et al., 2018), and accordingly, we adopted an approach to controlling for within-group effects (Turkeltaub et al., 2012); specifically, sets of activation coordinates from different contrasts, but the same participant group, were pooled. This means that, when we refer to the numbers of experiments, we have counted multiple contrasts from a single participant sample as one single experiment. In cases where two or more published articles contained data from the same participant sample, we pooled distinct contrasts as above, and excluded duplicates. This partially explains why the number of experiments in our analyses is lower than in those of some prior meta-analyses. However, in formal contrast analyses that compare different conditions (e.g., instructional cue, task difficulty), contrasts like these would be separated, and care was also taken to minimize the difference in the number of experiments on either side of the contrast. For example, if a study reported two contrasts - one implicit and one explicit based on the same participant group, only the peaks from the implicit task would be included in the contrast/conjunction analyses if there were a greater number of explicit than implicit tasks overall (see Fig. S9 of SI1).

(5) Only studies that tested healthy participants were included. Contrasts including clinical populations or pharmacological interventions were excluded.

(6) Only research articles published in English were included.

\subsubsection{Theory of mind}

This meta-analysis was built upon that of Molenberghs et al. (2016) and only included studies that were specifically designed to identify the neural network underpinning ToM processes (i.e., they employed tasks involving inferences about the mental states of others, including their beliefs, intentions, and desires). Therefore, studies that looked at passive observation of actions, social understanding, mimicry or imitation were not included, unless tasks included a ToM component. Unlike Molenberghs et al. (2016), we excluded studies investigating irony comprehension (e.g., Wang et al., 2006) because ToM might not always be necessary to detect nonliteral meaning in language (Ackerman, 1983; Bosco et al., 2018; Pexman, 2008) and studies that employed interactive games (e.g., Rilling et al., 2008). These latter studies are commonly designed to investigate the degree to which ToM is engaged under different task conditions rather than distinguish activation associated with ToM from that related to other processes. Moreover, unlike Molenberghs et al. (2016), we excluded studies that employed trait inference tasks as these were considered separately (see Section 2.1.3).

The Molenberghs et al. (2016) search was inclusive of fMRI studies published prior to July 2014 and yielded 144 independent experiments (1789 peaks) contributing to their analysis. We performed a WoS search for further original fMRI and PET studies conducted between August 2014 and March 2020, and a search of PET studies published prior to July 2014. We then applied our inclusion criteria to both newly identified studies and those analysed by Molenberghs and colleagues (see Table S1 of SI1 for further differences in criteria). In the end, we found 136 experiments with a total number of 2158 peaks and 3452 participants that met our criteria for inclusion (see Fig. S1of SI1 for more details regarding the literature selection process; and Table S1 of SI2 for a full list of the included experiments).

\subsubsection{Trait inference}

Studies were included in the meta-analysis if they employed tasks that required the participants to infer the personality traits of others based on prior person knowledge or another's appearance and/or behaviour. Whereas the types of mental states typically inferred in ToM tasks are transitory in nature (e.g., relating to immediate goals or the intentions behind a specific instance of behaviour), traits are coherent and enduring dispositional characteristics of others (i.e., personality traits; Van Overwalle, 2009). Previous meta-analyses (Molenberghs et al., 2016; Schurz et al., 2014) of ToM have included tasks requiring trait inferences. However, it has been suggested that personality trait inferences differ from mental state inferences in terms of likelihood and speed of processing, and hold a higher position in the hierarchical organisation of social inferential processes (Korman and Malle, 2016; Malle and Holbrook, 2012). In line with this proposal, we maintained a distinction and performed separate analyses. Moreover, previous imaging metaanalyses of trait inference were limited to studies that used face stimuli (Bzdok et al., 2011; Mende-Siedlecki et al., 2013). However, trait inferences can be made on the basis of many different sources of information, including physical appearance, behaviour and prior knowledge about others (Uleman et al., 2007). To our knowledge, the present attempt is the first to include studies that required participants to make trait inferences based on facial photographs, behavioural descriptions or prior person knowledge. We excluded any studies that asked participants to make inferences about transitory mental states, including basic emotions. We also excluded studies that did not use a subtraction approach, but rather investigated brain activity that varied parametrically with the levels of a pre-defined trait dimension (e.g. Engell et al., 2007). Finally, we excluded studies that included emotional face stimuli to avoid conflating brain activity related to trait inference with that associated with emotion recognition and processing. 
We performed a WoS search of studies published before March 2020 and reference-tracing to identify relevant studies for inclusion in the meta-analysis. A total of 40 experiments with 523 peaks and 732 participants were found to meet the criteria for inclusion (Fig. S2 of SI1; Table S2 of SI2).

\subsubsection{Empathy}

This meta-analysis was built upon that of Timmers et al. (2018) and only included studies that were specifically designed to identify the neural network underpinning empathy by employing tasks asking participants to observe, imagine, share and/or evaluate the emotional or sensory state of others. The task definition was kept identical to previous meta-analyses on empathy (Fan et al., 2011; Timmers et al., 2018). We also made a distinction between tasks eliciting empathic responses to other people's pain and those investigating empathic responses to others' affective states.

Timmers et al. (2018) included studies published before December 2017, totalling 128 studies with 179 contrasts (1963 peaks). We identified additional original studies conducted between January 2018 and March 2020 via a WoS search and subsequently applied our inclusion criteria to all, including those analysed by Timmers et al. (2018) (see Table S1 of SI1 for further differences in criteria). This resulted in a yield of 163 experiments with a total number of 2691 peaks and 4406 participants (Fig. S3 of SI1; Table S3 of SI2). Empathy for pain was independently investigated in 93 of these experiments, empathy for affective states was independently explored in 69 experiments, and 9 experiments concurrently explored both empathy for pain and emotions in the same contrasts.

\subsubsection{Moral reasoning}

This analysis updated a previous meta-analysis conducted by Eres et al. (2018) and included studies that employed tasks designed to investigate judgements and decision-making based on moral values. In line with Eres et al. (2018), studies that did not specifically have a morality component were not included. For example, studies investigating judgements regarding adherence to social expectations but not moral values (e.g., Bas-Hoogendam et al., 2017) were excluded.

Eres et al. (2018)'s search was restricted to fMRI studies and covered the period before February 2016 yielding 123 contrasts (989 peaks). We expanded this list via a WoS search for original fMRI and PET studies published between March 2016 and March 2020, and a search for PET studies published before March 2016, and then applied our inclusion criteria (see Table S1 of SI1 for differences in criteria). This resulted in a yield of 68 experiments with a total number of 884 foci and 1587 participants (Fig. S4 of SI1; Table S4 of SI2).

\subsubsection{Semantic control}

In this meta-analysis, we sought to extend an earlier meta-analysis conducted by Noonan et al. (2013) (also see Jackson, 2021). In line with theirs, this analysis only included studies that were specifically investigating semantic processing, and that reported contrasts that reflected high > low semantic control within a semantic task, or comparisons between a task requiring semantic control and an equally demanding executive decision in a non-semantic domain. We excluded studies with a focus upon priming without an explicit semantic judgement (e.g., primed lexical decision), bilingualism, episodic memory, or sleep consolidation.

Noonan et al. (2013)'s search covered the period between January 1994 and August 2009 and yielded 53 studies (395 peaks) that met their criteria for inclusion in their analysis. We performed a WoS search for original studies published between September 2009 and March 2020, and reference-tracing, and then applied our inclusion criteria to both newly identified studies and those analysed by Noonan et al. (2013). This produced a yield of 92 experiments with a total number of 971 peaks and 1966 participants that met the criteria for inclusion in our analysis (Fig. S5 of SI1; Table S5 of SI2).

\subsection{Data analysis}

We performed coordinate-based meta-analyses using the revised activation likelihood estimation (ALE) algorithm (Eickhoff et al., 2012, 2009; Turkeltaub et al., 2012) implemented in the GingerALE 3.0.2 software (http://brainmap.org/ale). We used the GingerALE software to conduct two types of analysis. The first were independent dataset analyses, which were used to identify areas of consistent activation across particular sets of experiments. These analyses were performed only on the experiment samples with a recommended minimum of 17 experiments in order to have sufficient power to detect consistent effects and circumvent the possibility of results being driven by single experiments (Eickhoff et al., 2016). The ALE meta-analytic method treats reported activation coordinates as the centre points of three-dimensional Gaussian probability distributions which take into account the sample size (Eickhoff et al., 2009). First, the spatial probability distributions of all coordinates reported were aggregated, creating a voxel-wise modelled activation (MA) map for each experiment. Then, the voxelwise union across the MA maps of all included experiments was computed, resulting in an ALE map that quantifies the convergence of results across experiments (Turkeltaub et al., 2012).The version of GingerALE used in the present study tests for above-chance convergence between experiments (Eickhoff et al., 2012) thus permitting random-effects inferences.

Following the recommendations of Müller et al. (2018), for the main statistical inferences, the individual ALE maps were thresholded using cluster-level family-wise error (FWE) correction of $p<.05$ with a prior cluster-forming threshold of $p<.001$ (uncorrected). Cluster-level FWE correction has been shown to offer the best compromise between sensitivity to detect true convergence and spatial specificity (Eickhoff et al., 2016). However, we subsequently applied an additional and more conservative threshold at the voxel level (FWE corrected at $p<.05$ ). This level of thresholding suffers from decreased sensitivity to true effects, but has the advantage of allowing an attribution of significance to each voxel and thereby increases the spatial specificity of inferences (Eickhoff et al., 2016). The FWE-corrected cluster-level and voxel-height thresholds were estimated using a permutation approach with 5000 repetitions (Eickhoff et al., 2012). None of the meta-analyses that we updated had used the recommended cluster-level FWE or the FWE heightbased correction methods.

The second set of analyses, conjunction and contrast analyses, were also performed in GingerALE and were aimed at identifying similarities and differences in neural activation between the different sets of studies. The conjunction images were generated using the voxel-wise minimum value (Nichols et al., 2005) of the included ALE maps to highlight shared activation. Contrast images were created by directly subtracting one ALE map from the other to highlight unique neural activation associated with each dataset (Eickhoff et al., 2011). Then, the differences in ALE scores were compared to a null-distribution estimated via a permutation approach with 5000 repetitions. The contrast maps were thresholded using an uncorrected cluster-forming threshold of $p<.001$ and a minimum cluster size of $200 \mathrm{~mm}^{3}$.

In addition, we performed post-hoc analyses to investigate if the clusters of convergence revealed by the ALE analyses were driven by experiments featuring specific characteristics of interest (i.e., type of instructional cue, task difficulty). To this end, we examined the list of experiments that contributed at least one peak to each ALE cluster and compared the number of contributing experiments featuring the characteristic of interest (e.g., explicit vs implicit processing) by conducting Fisher's exact tests of independence and post-hoc pairwise comparisons (using False Discovery Rate correction for multiple comparisons) in RStudio Version 1.2.5001 (RStudio Team, 2020).

A full list of the confirmatory and exploratory analyses we conducted can be found in Section S3 of SI1. 


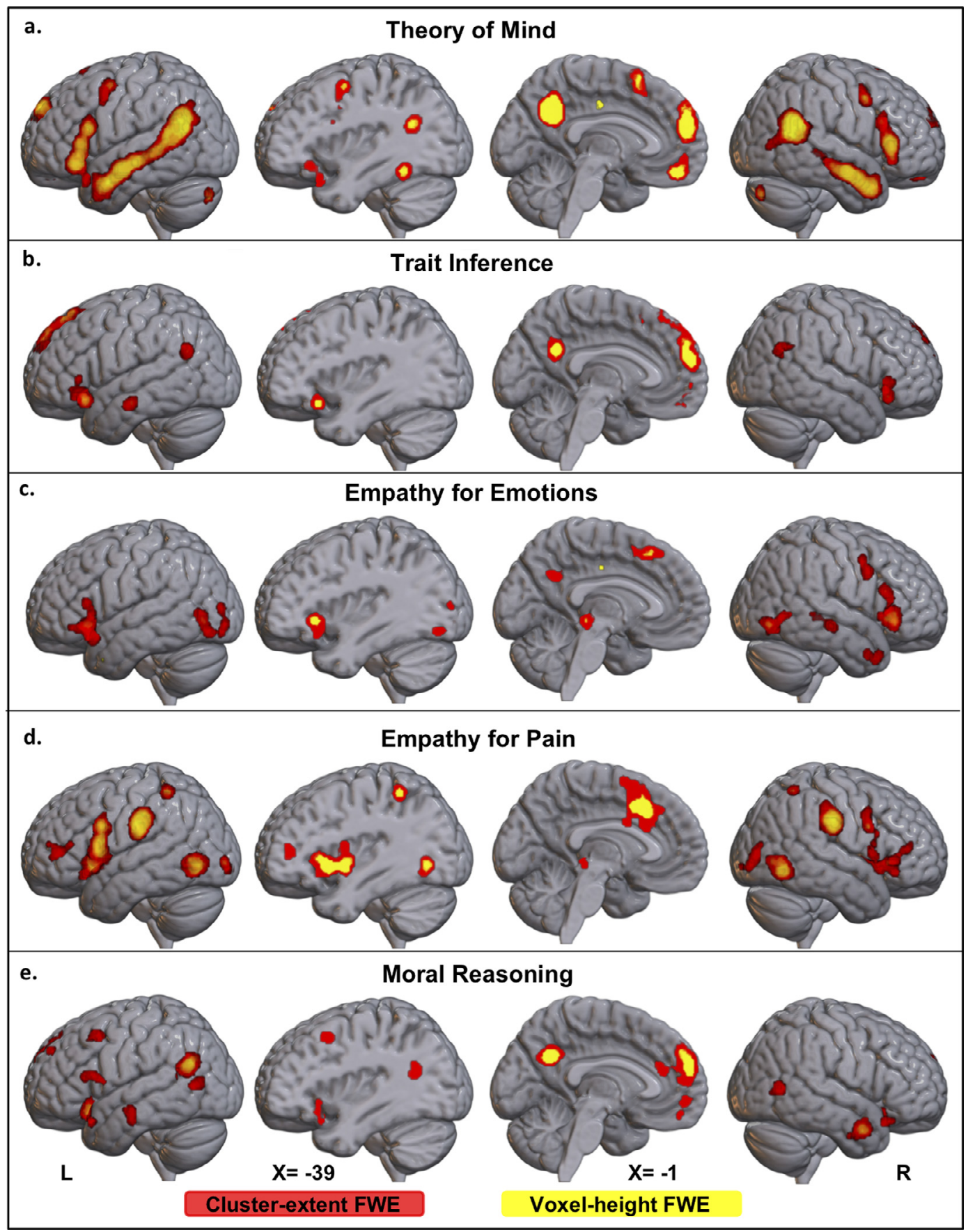

Fig. 1. Binary whole-brain ALE maps showing statistically significant convergent activation resulting from independent meta-analyses of ToM studies $(N=136)$, trait inference $(N=40)$, empathy for pain $(N=85)$ and emotions $(N=69)$ and moral reasoning $(N=68)$. The ALE maps were thresholded using an FWE corrected cluster-extent at $p<.05$ with a cluster-forming threshold of $p<.001$ (red) and an FWE corrected voxel-height threshold of $p<.05$ (yellow). The lateral views, which show projections on the cortical surface, are accompanied by brain slices at the sagittal midline and also coplanar with the peak of the left IFG cluster observed across all social domains $(X=-39$; Table S1.5).

\section{Results}

\subsection{The "Social brain"}

\subsubsection{Theory of mind}

Convergent activation across all 136 ToM experiments was found in 13 clusters (see Fig. 1a and Table S1.1.1 of SI3) located within the bilateral middle temporal gyrus (MTG) (extending anteriorly towards the temporal poles and also in a posterior and superior direction towards the superior temporal gyrus (STG) and angular gyrus (AG) in both hemi- spheres), bilateral IFG, bilateral dorsal precentral gyrus, ventromedial prefrontal cortex (vmPFC), dorsomedial prefrontal cortex (dmPFC), preSMA, precuneus, left fusiform gyrus and left and right cerebellum. All these clusters survived both the height-based and extent-based thresholding. A cluster in the posterior cingulate cortex (PCC) survived heightbased thresholding but did not survive extent-based thresholding. These results are largely consistent with those of Molenberghs et al. (2016), with the difference being that they did not find activation in SMA, left fusiform gyrus or cerebellum. In order to address concerns regarding the validity of some other popular ToM tasks (Heyes, 2014; Quesque and 
Rossetti, 2020), we conducted a separate supplementary meta-analysis that was limited to the subset of ToM experiments that employed false belief tasks (see Section S3.1 of SI1; Table S1.1.2). This analysis revealed convergent activation in similar temporo-parietal and medial frontal regions to the inclusive ToM analysis but did not implicate the lateral frontal cortex.

\subsubsection{Trait inference}

The ALE meta-analysis revealed convergent activation across 40 experiments in 8 clusters (Fig. 1b, Table S1.2) implicating the bilateral IFG, dmPFC, vmPFC, PCC, right pMTG (extending to AG), left AG and left anterior MTG. Voxels from all clusters, except for those in the right pMTG and vmPFC, survived the more conservative height-based thresholding.

\subsubsection{Empathy}

The ALE meta-analysis of all 163 empathy experiments revealed 16 clusters of convergent activation (Fig. S7a; Table S1.3.1), including in the bilateral IFG (extending towards the insula), SMA, dmPFC, bilateral posterior inferior temporal gyrus (ITG), right pMTG, bilateral supramarginal gyrus (SMG), left inferior parietal lobule (IPL), bilateral occipital cortex, bilateral amygdala, left thalamus, left caudate and brainstem. These clusters survived both the height-based and extentbased thresholding, except for the anterior $\mathrm{dmPFC}$ and right pMTG clusters which survived extent-based thresholding only. One cluster in the right cerebellum survived height-based thresholding but did not survive cluster extent-based thresholding. These areas were also implicated by Timmers et al. (2018). In contrast, however, we did not find convergent activation in the left posterior fusiform gyrus, left SMG (although we found a cluster slightly more posterior and inferior), left anterior ITG, right TP, precuneus, middle cingulate gyrus, and right superior parietal lobule.

The separate ALE maps for empathy for pain and empathy for affective states are displayed in Fig. $1 \mathrm{c}$ and d. A conjunction analysis found activation common to empathy for pain (Table S1.3.2) and empathy for affective states (Table S1.3.3) in the bilateral insula (extending to the IFG), SMA, right precentral gyrus, bilateral ITG, left occipital cortex and the brainstem (Fig. S7b; Table S1.3.4). Formal contrasts revealed that empathy for pain and empathy for emotions also engage highly distinct brain areas (Fig. S7b; Table S1.3.4). Clusters with increased convergence for empathy for pain were found in left IFG (pars triangularis), left precentral gyrus, bilateral insula, middle cingulate gyrus, bilateral SMG, right IPL and bilateral pITG. In contrast, increased convergence in empathy for affective states was revealed in PCC and right temporal pole. Given these significant differences in their underlying neural networks, empathy for pain and empathy for emotions were considered separately for all subsequent analyses.

\subsubsection{Moral reasoning}

Convergent activation across all 68 experiments studying moral reasoning was found in 12 clusters (Fig. 1e, Table S1.4) located in the left IFG, left insula (extending towards the superior temporal pole), right superior temporal pole (extending towards pars orbitalis of the IFG and insula), mPFC, medial orbitofrontal cortex (OFC), precuneus, bilateral pMTG, and the bilateral anterior MTG. Only four clusters - left insula, mPFC, precuneus and left pMTG - survived height-based thresholding. These results are mostly consistent with those obtained by Eres et al. (2018), with the difference that we did not find convergent activation in the left amygdala and right AG, and found additional clusters of convergent activation in left MFG, bilateral anterior MTG, and right pMTG.

\subsubsection{A common network for multiple sub-domains of social cognition}

To identify brain areas consistently activated across multiple subdomains of social cognition, we performed an overlay conjunction analysis of the cluster-extent FWE-corrected ALE maps associated with ToM, trait inference, empathy (for pain and/or emotions) and moral reasoning (see Fig. 2a, Table S1.5). Convergent activation across all four sociocognitive sub-domains was found in the left IFG (pars orbitalis) and precuneus. Overlapping areas of activation across three of four social sub-domains included right IFG, left IFG (pars triangularis and pars opercularis), SMA, mPFC, medial OFC, left MTG, left pMTG/AG, right anterior MTG and right PMTG/ITG. Overlap between two of four maps was found in bilateral precentral gyrus, right AG, right pMTG and left pMTG/ITG. Because the conservative thresholding used in this analysis could have excluded smaller clusters that nonetheless overlap across the sub-domains, we repeated the conjunction using ALE maps treated with a more liberal statistical threshold of $p<.001$ uncorrected. This revealed additional overlapping activation for all four social domains in the right IFG (pars orbitalis), mPFC, left pSTG/AG and bilateral ATL (Fig. S8). These brain areas have been implicated in a variety of social-cognitive abilities by multiple previous meta-analyses (Alcalá-López et al., 2018).

The extent to which brain regions engaged in social cognition overlap with those engaged in general semantic cognition (including both representation and control processes) is illustrated in Fig. 2b. Fig. 2c shows that the brain regions engaged in social cognition are largely non-overlapping with the network engaged by domain-general executive processes (i.e., the MDN).

\subsection{The semantic control network}

The ALE meta-analysis of all 92 semantic control experiments revealed convergent activation in a distributed network consisting of frontal, temporal and parietal areas (Fig. 3a, Table S2). The largest cluster was located in the left frontal lobe and extended from the IFG (pars orbitalis) to MFG. In the right frontal lobe, convergent activation was limited to two clusters with peaks in pars orbitalis and pars triangularis of the IFG. Consistent activation was also found in the medial frontal cortex with the peak in SMA. The left temporal cluster extended from the posterior portion of the MTG, which showed the highest level of convergence, to the fusiform gyrus. All these clusters survived both the height-based and extent-based thresholding. In addition, two left IPL clusters survived only the cluster-extent FWE correction. In contrast to Noonan et al. (2013), we did not find convergent activation in ACC, bilateral SFG, left AG, right IPL/SPL, and left anterior MTG.

The extent to which brain regions engaged in semantic control overlap with those engaged in general semantic cognition (including both representation and control processes), and domain-general executive processes (i.e., the MDN) are illustrated in Fig. 3 and is largely the same as that recently highlighted by Jackson (2021).

\subsection{Neural substrates shared by semantic control and social cognition}

\subsubsection{ToM}

Overlap between the neural network underpinning semantic control (i.e., SCN \& regions of the MDN) and the ToM network was found in 8 clusters located in the left IFG (including pars orbitalis and triangularis and extending to the precentral gyrus) and, to a smaller extent, the right IFG, the left dorsal precentral gyrus, SMA, left pMTG, left superior temporal pole and the left fusiform gyrus (Fig. 4a, Table S3.1.1). The results of the conjunction between semantic control and false belief reasoning can be found in Section S3.1 of SI1 and Table S3.1.2 of SI3. This analysis revealed overlapping activation in the PMTG, but not in the SMA or lateral frontal cortex.

\subsubsection{Trait inference}

Brain areas involved in both semantic control and trait inference included bilateral IFG (pars orbitalis), SMA and dmPFC (Fig. 4b, Table S3.2).

\subsubsection{Empathy for emotions}

The neural network underpinning semantic control overlapped with the areas engaged in empathy for emotions in bilateral IFG (pars orbitalis and pars triangularis) and SMA (Fig. 4c, Table S3.3). 


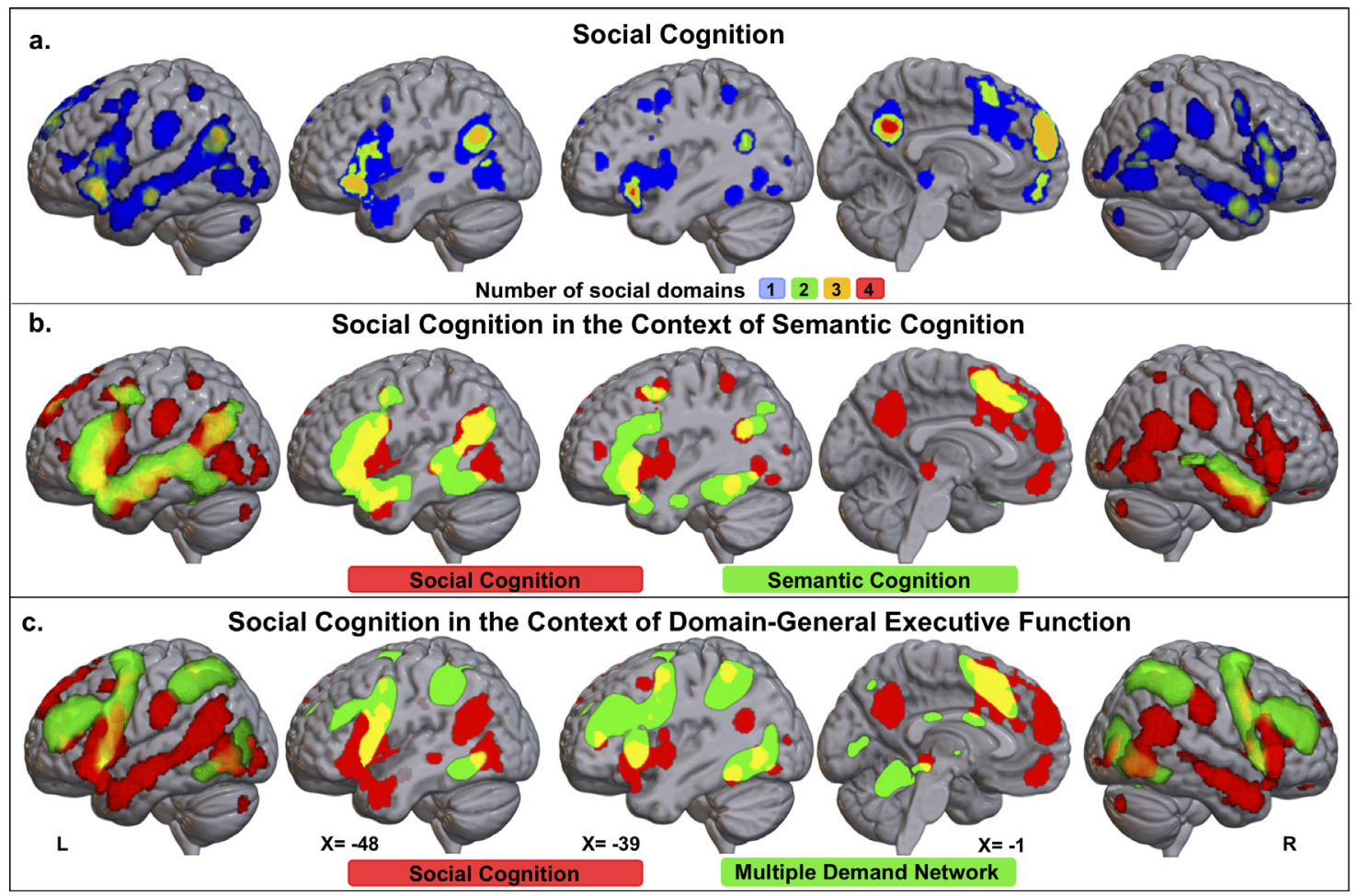

Fig. 2. The neural network engaged in social cognitive processing: (a) An overlay conjunction of the ALE maps resulting from independent meta-analyses of ToM studies, trait inference, empathy for pain/emotions, and moral reasoning. The map displays the number of social domains showing convergent activation in each voxel. The ALE maps were thresholded using an FWE corrected cluster-extent threshold at $p<.05$ with a cluster-forming threshold of $p<.001$. (b) The binarized social cognition map (red) generated by the overlay conjunction is displayed overlaid with a binarized ALE map of convergent activation across $N=415$ semantic $>$ non-semantic contrasts generated in Jackson, 2021 (green); overlap is shown in yellow. (c) The binarized social cognition map (red) generated by the overlay conjunction is displayed overlaid with a masque of the multiple-demand network (MDN) generated in Federenko et al., 2013 (green) by contrasting hard > easy versions of seven diverse cognitive tasks; overlap is shown in yellow. The lateral views, which show projections on the cortical surface, are accompanied by brain slices at the sagittal midline and also coplanar with the peak of the left STG $(X=-48)$ and left IFG $(X=-39)$ clusters that overlapped across all four social domains (Table S1.5).

\subsubsection{Empathy for pain}

Overlapping activation between empathy for pain and semantic control was revealed in left IFG (pars orbitalis and pars triangularis), right IFG (pars orbitalis), left precentral gyrus, bilateral insula, SMA and left posterior ITG (extending towards pMTG) (Fig. 4d, Table S3.4).

\subsubsection{Moral reasoning}

Overlapping activation in response to semantic control and moral reasoning included left insula (extending to pars orbitalis of the IFG), right IFG (pars orbitalis), left IFG (pars opercularis and pars triangularis), the left precentral gyrus and ACC (Fig. 4e, Table S3.5).

Overall, the neural network engaged in semantic control overlapped with the neural networks underpinning all four social domains in the bilateral IFG and, in particular, pars orbitalis. Except for moral reasoning, overlapping activation was also found in the SMA. In the left pMTG, we found a large area of overlap between semantic control and ToM and some evidence of overlap between semantic control and empathic processing.

\subsection{Explicit versus implicit social cognition}

Further to the meta-analyses above, we compared activation associated with implicit and explicit paradigms for studying empathy for emotions, empathy for pain and moral reasoning. The results of independent analyses are displayed in Fig. 5a-c and Tables S4.1.1-S4.1.6). Conjunctions and formal contrasts are displayed in Fig. 5D-f and Tables S4.2.1-S4.2.3). The only notable difference between activation associated with explicit and implicit paradigms, as identified by these formal comparisons, was in the case of empathy, with a small cluster in the
dmPFC showing increased convergence for explicit as compared to implicit empathy for pain (see Section S3.4.1.of SI1). In addition, we conducted exploratory cluster analyses to investigate whether the explicit and implicit experiments contributed similarly to each of the significant ALE clusters found for each social domain. In summary, these analyses (Fig. S9) revealed that in the case of all social domains, implicit and explicit experiments contributed equally to most clusters (see Section S3.4.2.of SI1 for a more detailed description).

\subsection{The relationship between cognitive effort and brain regions engaged during social cognitive tasks}

The above-reported conjunction analyses suggest that social cognition engages regions associated with semantic control. In these analyses, we took a pooled approach which involved collapsing over many different comparisons between social and non-social tasks and ignoring subtler differences between experimental and baseline conditions. The key advantage of this approach is that it identifies activation that is generalisable across highly variable experimental conditions. However, ignoring experimental differences precludes a determination of more specific factors driving a given region's involvement. In particular, it is not possible to directly infer from the above results that semantic control regions are specifically being engaged by the cognitive control demands of social tasks. Therefore, to address this issue, we performed a set of exploratory analyses to determine whether the IFG and PMTG regions are sensitive to the degree of cognitive effort required to complete social tasks. While these analyses cannot disentangle semantic control from other forms of control, they represent a further initial step towards confirming a role of semantic control regions in social regulatory processes. To this end, 


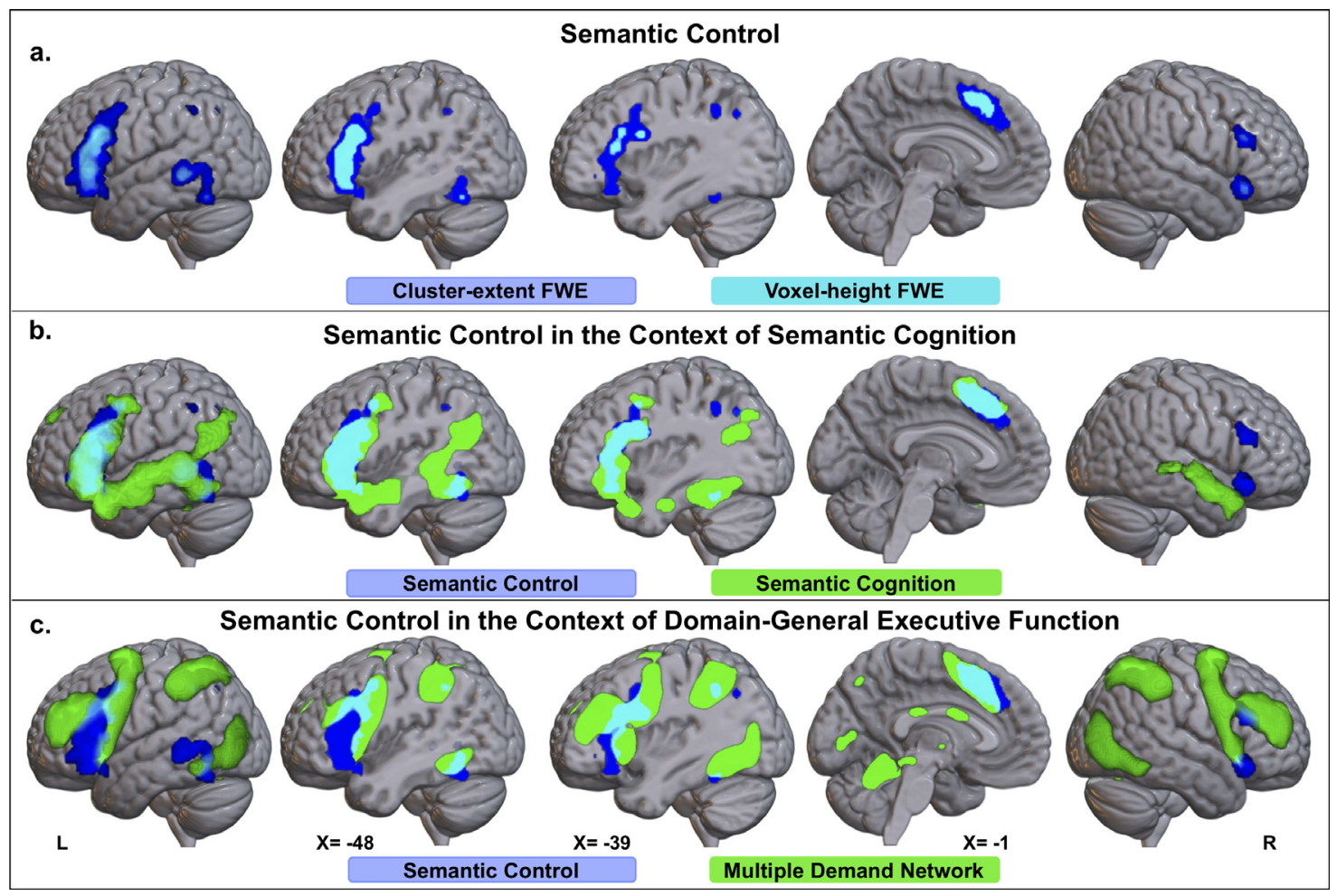

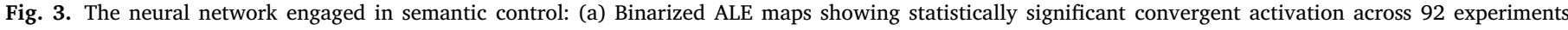

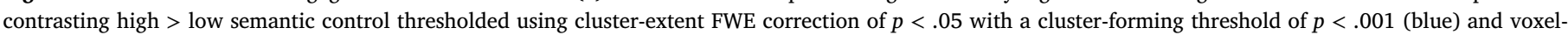

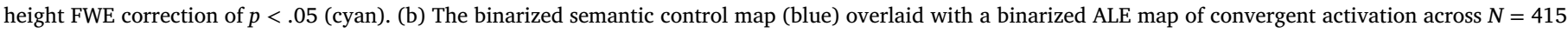

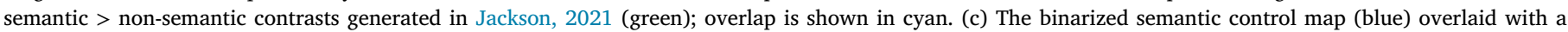

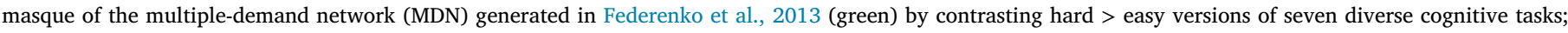

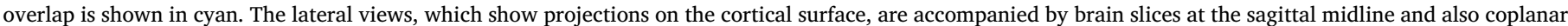
with the peak of the left STG $(X=-48)$ and left IFG $(X=-39)$ clusters that overlapped across all four social domains (Table S1.5).

we took experiments that used explicit paradigms and, on the basis of reported inferential statistics regarding participants' reaction/decision times, categorised them according to whether the experimental condition was more difficult than the control condition (E $>C$ ), experimental and control conditions were equally difficult $(\mathrm{E}=\mathrm{C})$, or the experimental condition was easier than the control condition $(\mathrm{C}>\mathrm{E})$. In the subsequent set of analyses we worked with the premise that in the case of $\mathrm{E}=\mathrm{C}$ experiments and $\mathrm{C}>\mathrm{E}$ experiments, activation associated with cognitive effort that is common to both the experimental and control conditions is subtracted away (along with activation specific to the control condition). In contrast, $\mathrm{E}>\mathrm{C}$ experiments preserve activation associated with cognitive effort that is specific to the experimental condition. Therefore, a contrast analysis pitting $\mathrm{E}>\mathrm{C}$ experiments against either $\mathrm{C}>\mathrm{E}$ or $\mathrm{E}=\mathrm{C}$ experiments will reveal activation associated with cognitive effort specific to the social domain. A conjunction will reveal activation associated with social processing irrespective of task difficulty.

There was only enough information regarding behavioural data to allow for sufficiently powered analyses in the case of ToM (Fig. S10 of SI1) where there were $26 \mathrm{E}>\mathrm{C}$ ToM experiments and $25 \mathrm{E}=\mathrm{C}$ ToM experiments. A conjunction analysis of $\mathrm{E}>\mathrm{C}$ and $\mathrm{E}=\mathrm{C}$ experiments yielded common activation in the left IFG (pars orbitalis and pars triangularis), dmPFC, precuneus, bilateral anterior MTG, right pMTG and left SMG (cyan in Fig. 6a; Table S5.3) which we interpret as regions engaged in ToM irrespective of task difficulty. Interestingly, a contrast of $\mathrm{E}>\mathrm{C}$ with $\mathrm{E}=\mathrm{C}$ ToM experiments revealed differential activation in the left pMTG, an area implicated in semantic control. The full reports of these analyses, including prerequisite independent ALE analyses on the $\mathrm{E}>\mathrm{C}$ ToM and $\mathrm{E}=\mathrm{C}$ ToM experiments, can be found in Tables S5.1-S5.4. For completeness, we also analysed $\mathrm{C}>\mathrm{E}$ ToM experiments, but the sample size $(N=14)$ was smaller than required to be sufficiently powered
(Eickhoff et al., 2016) and therefore the result should be interpreted with caution (Fig. 6a, Table S5.4).

Secondly, we conducted exploratory analyses to assess whether $\mathrm{E}>\mathrm{C}$, $\mathrm{E}=\mathrm{C}$ or $\mathrm{C}>\mathrm{E}$ ToM experiments were equally likely to contribute to each activation cluster (Fig. 6b). The clusters were identified in an independent ALE analysis of ToM experiments limited to those for which the behavioural information was known (Fig. 6c; Table S5.5). We expected clusters within brain areas that have a cognitive control function to have a disproportionate contribution from experiments in which the experimental task was more difficult than the control condition. To assess this, we conducted Fisher's exact tests and then interrogated significant main effects through post-hoc pairwise comparisons and using falsediscovery-rate adjustments for multiple comparisons. This cluster analysis revealed that $\mathrm{E}>\mathrm{C}, \mathrm{E}=\mathrm{C}$ and $\mathrm{C}>\mathrm{E}$ experiments contributed equally to $\mathrm{mPFC}(p=.67)$, precuneus $(p=.8)$, right anterior MTG $(p=.85)$, left pMTG $(p=.74)$, right pMTG $(p=.15)$ and right IFG $(p=.15)$. Contributions to the left IFG cluster depended on the difficulty category ( $p<$ .001 ) and pairwise comparisons indicated that the $\mathrm{C}>\mathrm{E}$ experiments contributed significantly less peaks compared to $\mathrm{E}>\mathrm{C}(p=.001)$ and $\mathrm{E}>\mathrm{C}$ ( $p=.046)$ experiments. Contributions to the left anterior MTG cluster also depended on the difficulty category $(p=.043)$ and pairwise comparisons indicated that the $\mathrm{C}>\mathrm{E}$ experiments contributed fewer peaks compared to $\mathrm{E}>\mathrm{C}$, but this effect did not survive correction for multiple comparisons $(p=.06)$. These results suggest that the left IFG is particularly sensitive to cognitively-challenging ToM processing.

\section{Discussion}

Although some contemporary theories of social cognition acknowledge the importance of control, or regulatory processes (Adolphs, 2010; 


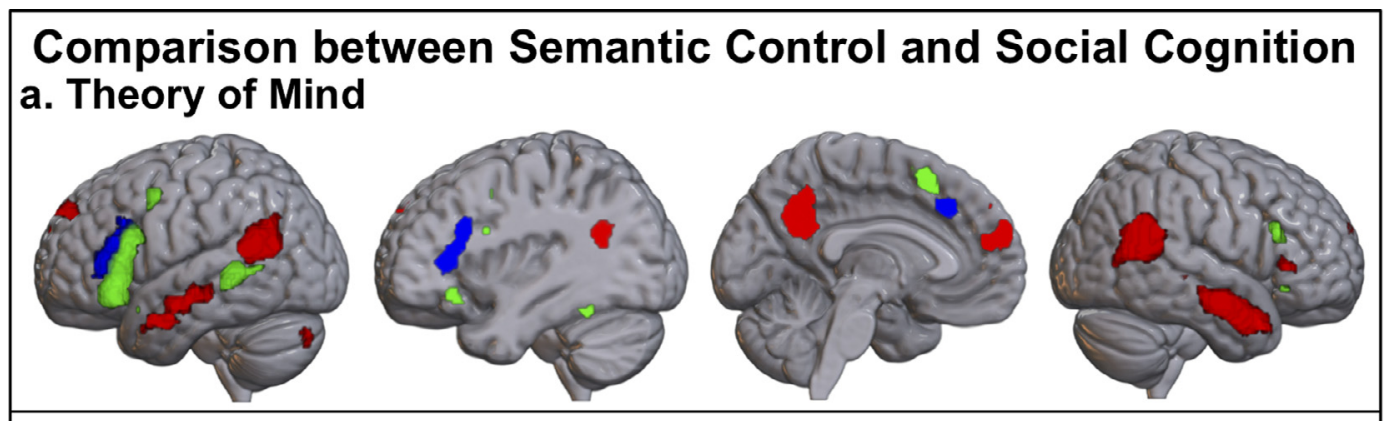

\section{b. Trait Inference}
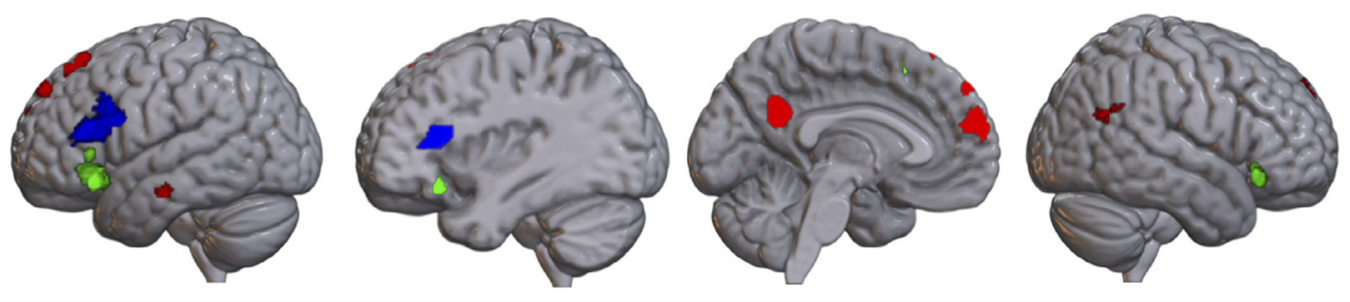

\section{c. Empathy for Emotions}
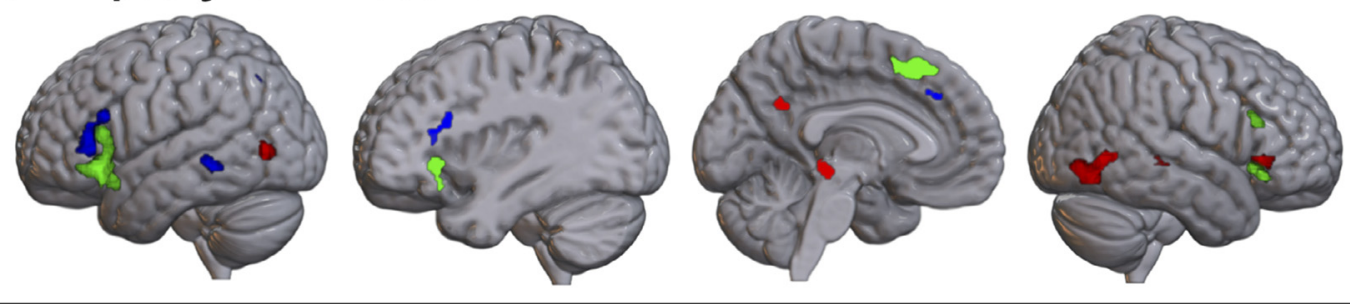

\section{d. Empathy for Pain}
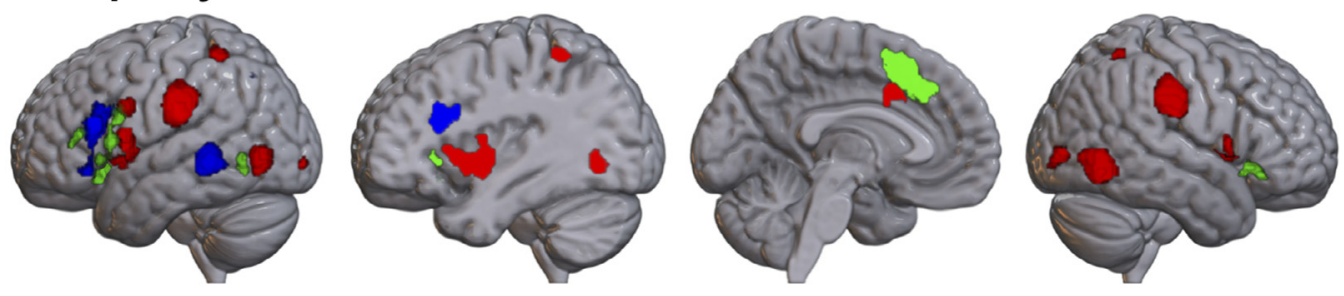

\section{e. Moral Reasoning}
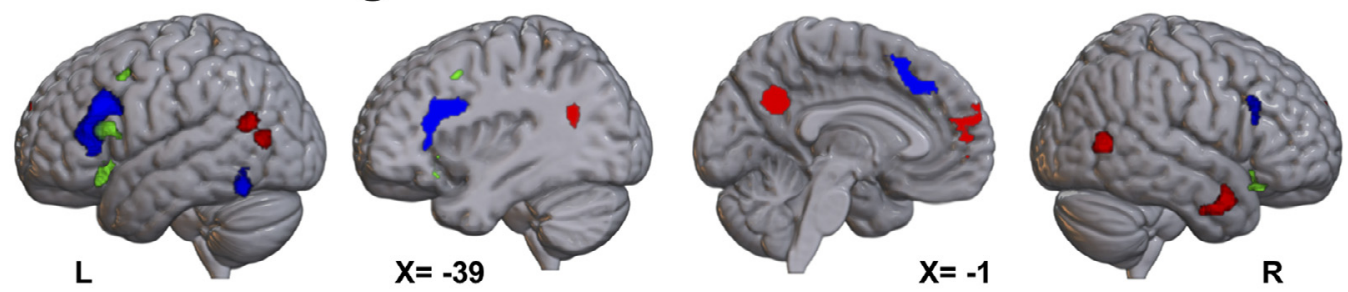

Semantic Control > Social

Conjunction

Social > Semantic Control

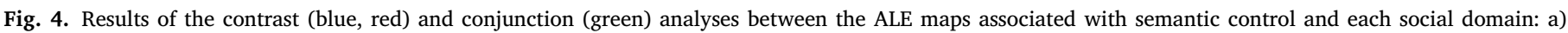

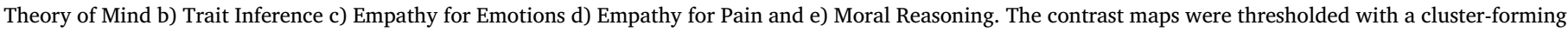

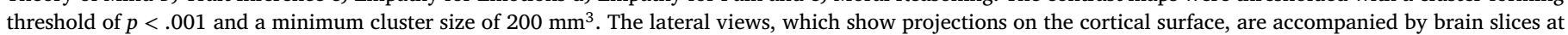
the sagittal midline and also coplanar with the peak of the left IFG cluster $(X=-39)$ that overlapped across all four social domains (Table S1.5).

Amodio and Cikara, 2021; Frith and Frith, 2012), many key questions remain about their exact nature and neural underpinnings. In the present study, we began to address three such questions: a) whether multiple forms of cognitive control contribute to social cognition, b) whether these control processes are ubiquitously involved in or selectively engaged by certain social abilities, and c) whether this engagement is dependant on specific task demands (e.g. instructional cue) (Binney and
Ramsey, 2020). Specifically, we set out to investigate whether brain regions implicated in the controlled retrieval and selection of conceptual knowledge - particularly the IFG and pMTG comprising the SCN (Jefferies, 2013; Lambon Ralph et al., 2017) - contribute to social processing. We simultaneously applied this question to multiple sub-domains of social cognition so that we could assess the extent to which involvement is general, or specific to certain types of social tasks 


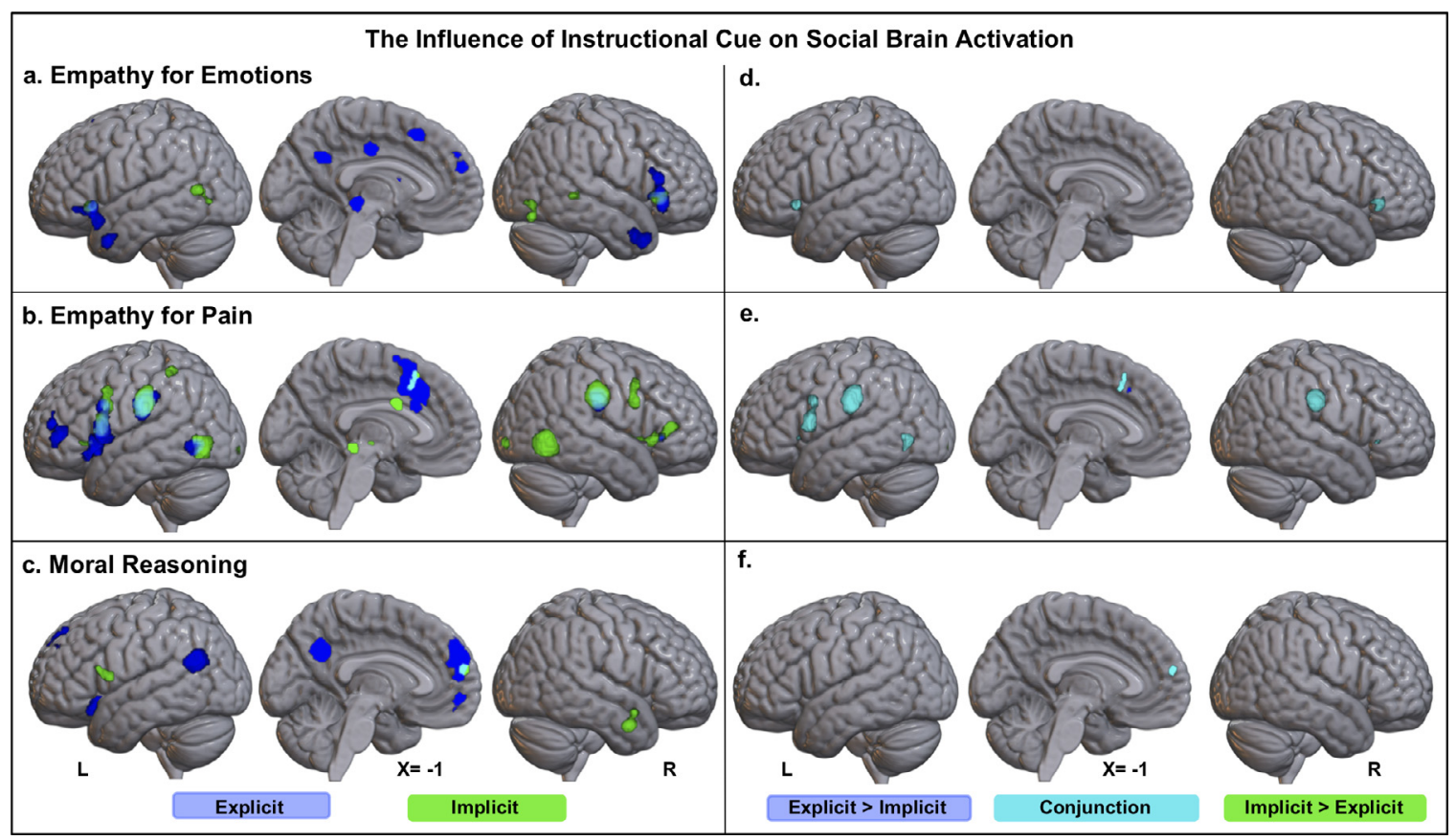

Fig. 5. The left panel displays the binary ALE maps showing statistically significant convergent activation resulting from independent meta-analyses on explicit (blue) and implicit (green) studies on a) Empathy for Emotions, b) Empathy for Pain and c) Moral Reasoning. The ALE maps were thresholded using an FWE corrected cluster-extent threshold of $p<.05$ with a cluster-forming threshold of $p<.001$. The right panel displays the results of the contrast (dark blue, green) and conjunction (cyan) analyses between the ALE maps associated with explicit and implicit instructions. The contrast maps were thresholded at $p<.001$ and using a minimum cluster size of $200 \mathrm{~mm}^{3}$. The lateral views, which show projections on the cortical surface, are accompanied by brain slices at the sagittal midline.

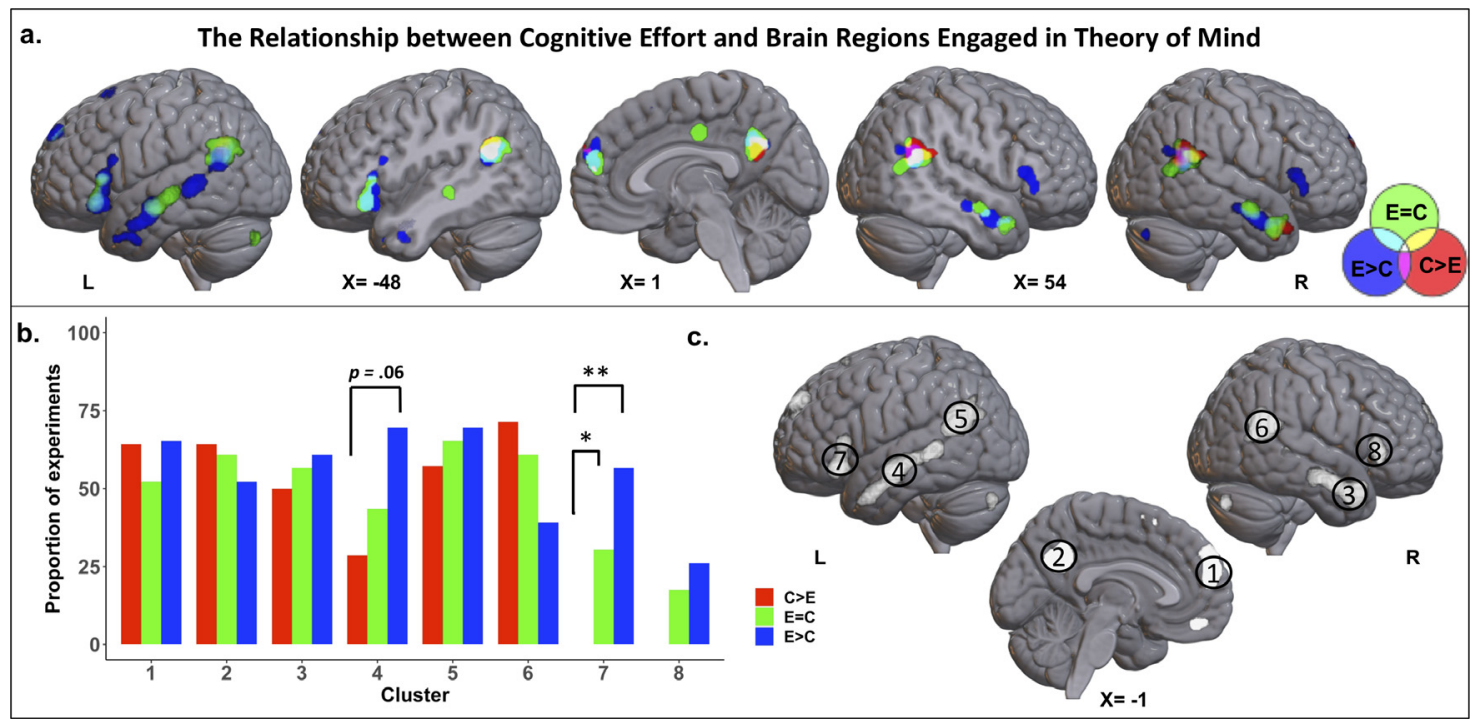

Fig. 6. Results of exploratory analyses investigating the effect of task difficulty on ToM activation: (a) Binary ALE maps showing statistically significant convergent activation resulting from independent meta-analyses of three subsets of explicit ToM studies characterized by experimental conditions that were harder than the control task (E>C; $N=26$; blue), experimental and control conditions that were equally difficult (E=C; $N=27$; green) and control conditions that were harder than the experimental condition (C>E; $N=14$; red) as indexed by participant reaction times. The ALE maps were thresholded using an FWE corrected cluster-extent threshold at $p<.05$ with a cluster-forming threshold of $p<.001$. The lateral views, which show projections on the cortical surface, are accompanied by brain slices at the sagittal midline and also coplanar with the peak of the left IFG cluster $(X=-39)$ that overlapped across all four social domains (Table S1.5) and the right pSTG cluster from the ToM meta-analysis (Table S1.1.1). (b) The results of the cluster analyses where bars represent the proportion of experiments in each difficulty category contributing to clusters of interest resulting from an ALE analysis of $N=60$ ToM meta-analysis which included E $>\mathrm{C}, \mathrm{E}=\mathrm{C}$ and $\mathrm{C}>\mathrm{E}$ experiments. (c) Binary ALE map showing statistically significant convergent activation across ToM experiments limited to those for which the behavioral information was known - this map represented the basis of the cluster analysis. The ALE map was thresholded using an FWE corrected cluster-extent threshold at $p<.05$ with a cluster-forming threshold of $p<.001 ;{ }^{* *} p<.001 * p<.05$. 
and/or abilities. And we adopted a formal meta-analytic approach to extracting reliable trends from across a large number of functional neuroimaging studies and overcome the limitations of individual experiments (Cumming, 2014; Eickhoff et al., 2012). We found that theory of mind, trait inference, empathy, and moral reasoning commonly engage a core social network that includes the left IFG, precuneus and, when more liberal thresholds are applied, the right IFG, mPFC, bilateral ATL and left pMTG/AG. Moreover, the IFG (particularly the pars orbitalis) region greatly overlapped with that implicated in an independent meta-analysis of neuroimaging studies of semantic control. Further, exploratory analyses suggest that both the left anterior IFG and the left posterior MTG (at a position just anterior to the 'temporoparietal junction') are sensitive to executive demands of social tasks. We interpret our overall findings as supportive of the hypothesis that the SCN supports social cognition via a process of controlled retrieval of conceptual knowledge. This aligns with a broader proposal in which social cognition is described as a flavour of domain-general semantic cognition and relies on the same basic cognitive and brain systems (Binney and Ramsey, 2020).

\subsection{Cognitive control in social cognition}

\subsubsection{The contribution of semantic control}

Alternative theories and existing findings regarding cognitive control in social cognition point to distinguishable mechanisms for monitoring conflict and errors, and for implementing or inhibiting responses (see, for example, Amodio, 2014). However, the exact nature of the information or processes being controlled is not clear. Involvement of the SCN in social cognition suggests that it is, at least in part, related to a controlled attribution of meaning to stimuli and experiences, and to the production of task-appropriate meaning-imbued behaviour (Corbett et al., 2015; Lambon Ralph et al., 2017). Within the broader literature regarding semantic control, a key distinction has been drawn between a) top-down goal-directed retrieval and b) post-retrieval selection of goal-relevant semantic knowledge (Badre et al., 2005; Jefferies, 2013; ThompsonSchill et al., 1997), and it has been suggested that both of these two control mechanisms contribute significantly to interpersonal interactions (Binney and Ramsey, 2020; Satpute and Lieberman, 2006). Studies of semantic cognition suggest that 'selection' is engaged when bottom-up, automatic activation of conceptual knowledge results in multiple competing semantic representations and/or responses. Social interactions frequently involve subtle or ambiguous cues, such as neutral facial expressions and bodily gestures, and/or conflicting cues (e.g., sarcasm). This causes semantic competition that can only be resolved by taking into account the wider situational and linguistic context and/or prior knowledge about the speaker (Aviezer et al., 2008; Pexman, 2008). Controlled retrieval processes, on the other hand, are engaged when automatic semantic retrieval fails to activate the semantic information necessary for the task at hand. This may occur frequently in social interactions, and particularly with less familiar persons, because of a preponderance of surface features (e.g., physical characteristics) over less salient features (e.g., personality traits, preferences, and mental states). To avoid exchanges that are deemed superficial at best, controlled retrieval must be used to bring to the fore person-specific but also context-relevant semantic information. On the basis of observations in other domains, it is possible to make some predictions about what social behaviour might look like when these semantic regulatory processes fail. For example, semantic errors in language production (as opposed to phonological errors, for example) arise because of demanding (e.g., speeded) testing conditions, as well as brain pathology (Hodgson and Lambon Ralph, 2008; Jefferies and Lambon Ralph, 2006), and can be categorised according to three types: superordinate (e.g., saying "animal" in response to a picture of a horse), coordinate (e.g., saying the name of an incorrect but related, often more familiar concept, e.g., "cat") and associative errors (e.g., "stable"). These errors reflect a failure to access the correct meaning associated with a stimulus. When the ability to impose semantic con- trol is compromised during social interactions one might observe similar types of errors; that is behavioural responses that are incongruous with, albeit distantly semantically related to incoming interpersonal signals. Further, one would predict that these errors are less likely when contextual anchors constrain the possible meanings and reduce the reliance on semantic control. In line with this, a recent study has demonstrated effects of impaired semantic control on emotion perception (Souter et al., 2021).

There is now over a decade's worth of multi-method evidence that semantic control is underpinned by the left IFG and the left pMTG (Jefferies, 2013; Lambon Ralph et al., 2017). Research is now aimed at understanding the neural mechanisms by which these regions modulate semantic processing. One recent proposal is that it involves coordination of spreading activation across the semantic representational system (Chiou et al., 2018). According to the hub-and-spoke theory of semantic representation (Lambon Ralph et al., 2017), coherent concepts are represented conjointly by a central supramodal semantic 'hub' located in the ATLs, as well as multiple distributed areas of association cortex (i.e. 'spokes') that represent modality-specific information (e.g. visual features, auditory features, verbal labels, etc.). Chiou et al. (2018) showed that the left IFG could be imposing cognitive control by flexibly changing its effective connectivity with the hub and spoke regions according to task characteristics; the IFG displayed enhanced functional connectivity with the 'spoke' region that processes the most task-relevant information modality. A similar proposal has been made for the contribution of domain-general cognitive control systems to social information processing. Zaki et al. (2010) found that, in the presence of conflicting social cues, right IFG activity becomes functionally coupled with the brain areas associated with processing the particular cue type the participant chose to rely on to make inferences about emotional states. On this basis, they proposed that cognitive control areas upregulate activation within systems that represent social cues that are currently most relevant to the task. Consistent with this, a further study found evidence to suggest that the left IFG downregulates neural activation associated with taskirrelevant self-referential information when the task required reference to others (and vice versa) (Soch et al., 2017).

An important feature of semantic processing is the ability to accommodate new information that emerges over extended periods of time and update our internal representation of the current "state of affairs" in the external world according to contextual changes. This is particularly important for navigating social dynamics which are liable to abrupt and sometimes extreme changes in tone. For instance, imagine being in a bar and having your attention drawn to someone standing suddenly and picking up a glass. One might reasonably infer that this person is thirsty. That is until they proceed to walk towards a group of noisy sports fans rather than the bartender. In this case, you will likely adapt your interpretation and engage in a pre-emptive defensive stance. Recent research suggests that this ability to update depends, at least in part, on the IFG, as well as the MPFC and ventral IPL (also see Section 4.2.2) (Branzi et al., 2020). Likewise, Lavoie et al. (2016) showed that, during a ToM task, activation of the left IFG and pMTG is associated with contextual adjustments of mental state inferences (and also more general physical inferences) although not the representation of mental states specifically. Left IFG activation has also been observed when newly-presented information requires one to update the initial impression formed of another person (e.g., Mende-Siedlecki et al., 2013b, 2013a; Mende-Siedlecki and Todorov, 2016).

\subsubsection{The wider contribution of executive processes}

According to Lambon Ralph, Jefferies, and colleagues, the executive component of semantic cognition comprises both semantic control and other domain-general processes (Lambon Ralph et al., 2017; Binney and Ramsey, 2020). The latter includes top-down attentional control and working memory systems that support goal-driven behaviour irrespective of the task domain (i.e., perceptual, motor or semantic). These processes recruit nodes of the MDN (Duncan, 2010), which include the pre- 
central gyrus, MFG, IPS, insular cortex, pre-SMA and adjacent cingulate cortex (Assem et al., 2020; Fedorenko et al., 2013). In terms of organisation, the SCN appears to be nested amongst domain-general executive systems (Wang et al., 2020) and could play a role in mediating interactions between the MDN and the semantic representational system (Davey et al., 2016; Lambon Ralph et al., 2017). In line with this general perspective, we expected MDN regions to be reliably engaged by all four social sub-domains explored in the present meta-analyses. While there was evidence of engagement of the MFG, the pre-SMA, ACC, insula and IPS in some of the social sub-domains, MDN regions were not part of the core social processing network identified by the overlay conjunction analysis. This could reflect the fact that the majority of contrasts included in our meta-analyses employed high-level control conditions that were well-matched to the experimental conditions in terms of general task requirements, and thus, most activation associated with general cognitive demands had been subtracted away. Consistent with this notion is the fact that studies contrasting social tasks with lower-level control conditions (e.g., passive fixation) find more extensive MDN activation in ToM (Mason et al., 2008; Mier et al., 2010), trait inference (Chen et al., 2010; Hall et al., 2012), empathy (De Greck et al., 2012; Tamm et al., 2017) and moral reasoning (Reniers et al., 2012). The role of the MDN in social cognition is otherwise becoming well-established, and it has been found to be sensitive to difficulty manipulations in social tasks, showing increased activation in response to conflicting social cues (Cassidy and Gutchess, 2015; Mitchell, 2013), social stimuli that violate expectations (Cloutier et al., 2011; Hehman et al., 2014; Ma et al., 2012; Weissman et al., 2008) and increasing social working memory load (Meyer et al., 2012).

Finally, it is important to note that, although both MDN and the SCN co-activate in social and semantic tasks, the nature of their specific contributions and their anatomy are at least partially dissociable. The MDN is associated with the implementation of top-down constraints to facilitate goal-driven aspects of processing that is not limited to the semantic domain (Duncan, 2013; Fedorenko et al., 2013; Whitney et al., 2012). In contrast, the engagement of the anterior ventrolateral IFG (pars orbitalis) and the left pMTG appear specific to the semantic domain and, in particular, controlled semantic retrieval (Badre and Wagner, 2007; Dobbins and Wagner, 2005; Whitney et al., 2012). Unlike the MDN, they do not appear to respond to challenging non-semantic tasks (Gao et al., 2020; Hodgson et al., 2021; Noonan et al., 2013; Whitney et al., 2012). Further, tasks associated with low conceptual retrieval demands but a requirement for response inhibition engage the MDN but do not engage the SCN, even if conceptual knowledge is used to guide responses (Alam et al., 2018).

\subsubsection{Double-route vs single-route cognitive architecture of social cognition}

A secondary aim of the present study was to address a pervasive distinction in the social neuroscientific literature between automatic and controlled processes (Adolphs, 2010; Happé et al., 2017; Lieberman, 2007). Some authors have argued that automatic and controlled social processes are mutually exclusive of one another and draw upon distinct cortical networks (Forbes and Grafman, 2013; Lieberman, 2007; Satpute and Lieberman, 2006). The alternative is a single-route architecture where the degree to which behaviours have particular attributes (e.g. speed, effort, intentionality) does not reflect the involvement of one system and not another, but quantitative differences in the extent to which the control system interacts with the representational system in order to produce context-/task- appropriate responses (Binney and Ramsey, 2020). Our results are consistent with the latter perspective. The brain regions reliably activated in response to explicit instructions and those associated with implicit instructions revealed more overlap than discrepancy across empathy and moral reasoning tasks. Notably, this overlap included brain areas associated with executive functions: the bilateral IFG in the case of empathy for emotions and bilateral IFG and AmPFC in the case of empathy for pain. Moreover, cluster analyses of the ALE maps associated with the four so- cial domains suggest that studies using explicit and implicit paradigms (which are assumed to engage controlled and automatic processing respectively) contributed equally to most activation clusters, including those in brain regions associated with control processes. Contrary to the predictions of dual-process models, these findings suggest that common neural networks contribute to both explicit and implicit social processing (also see Van Overwalle and Vandekerckhove, 2013). Furthermore, exploratory analyses suggest that both the left anterior IFG and the pMTG are sensitive to executive demands of social tasks. Overall, we argue that these results support the existence of a single-route cognitive architecture wherein the contribution made by control mechanisms to implicit and explicit social processing reflects cognitive effort demanded by the task at hand. This follows similar proposals put forth specifically in the domain of ToM (Carruthers, 2017, 2016).

\subsection{Beyond cognitive control}

Our findings converged upon four further regions that have been strongly linked with key roles in social cognition: the mPFC (including the anterior cingulate), the precuneus, the 'temporoparietal junction' (TPJ), and the ATL. We briefly discuss the putative role of each of these regions below.

\subsubsection{The 'Temporo-parietal junction'}

A region often referred to as the 'temporo-parietal junction' (TPJ) has been subject to an elevated status within the social neurosciences. In particular, the right TPJ has been attributed with a key role in representing the mental states of others (Saxe and Wexler, 2005). In line with previous meta-analyses (Bzdok et al., 2012; Molenberghs et al., 2016; Schurz et al., 2020, 2014, 2013), our results reveal a bilateral TPJ region that is reliably involved in ToM tasks. In the left hemisphere, an overlapping area is also implicated in trait inference, moral reasoning and, when a more lenient threshold was applied, empathy for emotions, which is suggestive of a broader role of the left TPJ in social cognition. In contrast, the right TPJ showed more limited overlap, being reliably engaged only by ToM and trait inference tasks, which is suggestive of a more selective role of the right TPJ in social cognition.

The TPJ encompasses a large area of cortex that is poorly defined anatomically and seems to include parts of the AG, SMG, STG and MTG (Schurz et al., 2017). Moreover, this area is functionally heterogeneous and has been associated with a variety of cognitive domains including but not limited to attention, language, numerosity, episodic memory, semantic cognition and social perception (Binder et al., 2009; Decety and Lamm, 2007; Deen et al., 2015; Humphreys and Lambon Ralph, 2015a; Igelström and Graziano, 2017; Özdem et al., 2017; Quadflieg and Koldewyn, 2017). While there is some indication that the function of the TPJ may be dependant on the hemisphere (e.g., Numssen et al., 2021), many cognitive domains, including ToM, are associated with bilateral TPJ activation. Our results at least seem to suggest dissociable roles of pMTG and a more posterior TPJ region; while the left pMTG is activated within both semantic control and ToM studies, a separate and more posterior STG (TPJ) area located closer to SMG/AG was reliably engaged by three of the social domains, but not studies of semantic control. Furthermore, the results suggest that the left pMTG is sensitive to the difficulty of ToM tasks while the bilateral pSTG (TPJ) region is not.

This finding is generally in line with previous research suggesting a functional dissociation between the left pMTG and the left ventral IPL/AG regions. From one perspective, the activation of both regions appears to be positively associated with semantic tasks (Binder et al., 2009). However, the left pMTG shows increased activation to difficult relative to easier semantic tasks (Jackson, 2021; Noonan et al., 2013), unlike the ventral IPL/AG which has been shown to deactivate to semantic tasks when they are contrasted against passive/resting conditions where there may be greater opportunity for spontaneous semantic processing or 'mind-wandering' (Humphreys et al., 2015; Humphreys and Lambon Ralph, 2015b). Moreover, Davey et al. (2015) found that TMS 
applied to pMTG disrupted processing of weak semantic associations more than for strong associations, whereas TMS applied to AG had the opposite effect. Based on these and similar observations it has been suggested that the ventral IPL/AG has a role in the automatic retrieval of semantic information.

\subsubsection{The default mode network}

The pSTG/AG, the mPFC and precuneus regions we identified as part of the core social cognition network are also considered part of the default-mode network (DMN) (Buckner et al., 2008; Spreng and Andrews-Hanna, 2015). The DMN is a resting-state network, meaning that it is a group of regions consistently co-activated without the requirement of an explicit task. It is proposed that it is ideally suited for supporting self-generated internally-orientated, as opposed to externallyorientated, cognition (i.e., it is decoupled from sensory processing; Margulies et al., 2016; Smallwood et al., 2013). Some of these regions (e.g., the AG and $\mathrm{mPFC}$ ) have also been implicated in processes that allow the integration of information over time (Huey et al., 2006; Humphreys et al., 2020; Ramanan et al., 2018; Ramanan and Bellana, 2019). These purported functions are all presumably important for social and more general semantic processing (see Section 4.1.1.) and likely involve domain-general mechanisms (also see Van Overwalle, 2009). However, the degree to which regions implicated in the DMN and those implicated in social and/or semantic cognition do or do not overlap is contentious and much is left to be gleaned regarding the relationship between these systems (Jackson et al., 2021, 2019; Mars et al., 2012).

\subsubsection{The anterior temporal lobe}

Our findings implicate the lateral anterior temporal lobe (ATL), and particularly the dorsolateral STG/temporal pole (BA 38) and middle anterior MTG/STS, in all the socio-cognitive domains investigated, except for empathy for pain. Exploratory cluster analyses revealed that ATL engagement is not dependant on instructional cue or task difficulty, and thus it appears to serve a role other than control.

A key contribution of the ATL to social-affective behaviour has been recognised by comparative and behavioural neurologists for well over a century, owed at first to the acclaimed work of Brown and Schafer (1888) and, later, Klüver and Bucy (1939) who provide detailed reports of profound social and affective disturbances in non-human primates following a bilateral, full depth ATL resection. These observations are mirrored in descriptions of neurogenerative patients that associate progressive ATL damage with a wide range of socio-affective deficits (Binney et al., 2016; Chan et al., 2009; Ding et al., 2020; Perry et al., 2001), including impaired emotion recognition (Lindquist et al., 2014; Rosen et al., 2004) and empathy (Rankin et al., 2005), impaired capacity for ToM (Duval et al., 2012; Irish et al., 2014), and a loss of person-specific knowledge (Gefen et al., 2013; Snowden et al., 2012, 2004). Over the past 10 years, there been a growing acceptance of the central role played by the ATL within the social neurosciences (Olson et al., 2013) and it now features prominently in some neurobiological models of face processing (Collins and Olson, 2014), ToM (Frith and Frith, 2006), moral cognition (Moll et al., 2005), and emotion processing (Lindquist et al., 2012). It has also been pinpointed as a key source of top-down influence on social perception (Freeman and Johnson, 2016). One influential account of social ATL function proposes a domain-specific role in the representation of social knowledge, including person knowledge, and other more abstract social concepts (Olson et al., 2013; Thompson et al., 2003; Zahn et al., 2007).

A parallel line of research focused upon general semantic cognition has given rise to an alternative, more domain-general account of ATL function; there is a large body of convergent multi-method evidence from patient and neurotypical populations in support of a role of the ATL in the formation and storage of all manner of conceptual-level knowledge (Lambon Ralph et al., 2017). Research efforts have therefore recently begun to ask whether the purported roles of the ATL in both social and semantic processes can be reconciled under a single unifying framework (Binney et al., 2016b; Rice et al., 2018). Some clues already exist in the aforementioned work of Klüver and Bucy (1939), who observed a broader symptom complex comprising multimodal semantic impairments, including visual and auditory associative agnosias, that might explain rather than just co-present with social-affective disturbances. More recent work that leverages the higher spatial resolution of functional neuroimaging in humans has revealed a ventrolateral ATL region that responds equally to all types of concepts, including social, object and abstract concepts, be they referenced by verbal and/or non-verbal stimuli (Binney et al., 2016b; Rice et al., 2018). Activation of the dorsal-polar ATL, on the other hand, appears to be more sensitive to socially-relevant semantic stimuli (Binney et al., 2016b; Rice et al., 2018; Zahn et al., 2007). These observations support a proposal in which the broadly-defined ATL region can be characterised as a domain-general supramodal semantic hub with graded differences in relative specialisation towards certain types of conceptual information (Binney et al., 2012; Binney et al., 2016b; Lambon Ralph et al., 2017; Plaut, 2002; Rice et al., 2015). Our results reveal that the temporal poles are reliably activated across four social domains - moral reasoning, empathy for emotions, ToM and trait inference. They do not, however, provide support for the involvement of the ventrolateral ATL. We argue this is likely due to technical and methodological limitations of the fMRI studies included in the meta-analyses (see Visser et al., 2010). Most notably this includes vulnerability to susceptibility artefacts that cause BOLD signal drop-out and geometric distortions around certain brain areas, including the ventral ATLs (Jezzard and Clare, 1999; Ojemann et al., 1997). Studies that have used PET, which is not vulnerable to such artefacts, or techniques devised to overcome limitations of conventional fMRI (Devlin et al., 2000; Embleton et al., 2010), reveal activation in both the temporal poles and the ventral ATL in response to social stimuli (Balgova et al., 2021; Binney et al., 2016b; Castelli et al., 2002).

\subsection{Limitations}

Because semantic control demands were not explicitly manipulated in the social contrasts we included, our results cannot directly confirm our hypothesis regarding the specific contribution made by the SCN in social cognition. Our conclusions rely on an assumption that overlap reflects a generalised neurocomputation upon which both semantic control and social processing rely. The alternative explanation is that overlapping activation reflects tightly yet separately packed cognitive functions which may only dissociate when investigated at an increased spatial resolution (Henson, 2006; Humphreys et al., 2020). Moreover, we chose to pool across heterogeneous samples of studies to investigate the cognitive domains of interest. The advantage of this approach is that it identifies activation that is generalisable across highly variable experimental conditions and washes out spurious findings associated with idiosyncratic properties of stimuli and/or paradigms. However, the preponderance of specific experimental procedures in each literature addressed still unintentionally led to systematic differences in the characteristics of the studies used to define the different cognitive domains. For example, the semantic control dataset included studies that employed verbal stimuli almost exclusively, while the majority of empathy studies employed non-verbal stimuli. Some of the differences between the associated networks (e.g., in lateralization) might therefore be attributable to verbal processing demands. As is the case with all meta-analyses, therefore, some aspects of our results should be treated with caution.

Another limitation of this study is that most of the experiments included used control conditions that were highly matched to their experimental conditions in terms of the demand for domain-general processes such as cognitive control and semantic processing, and therefore they may have subtracted away much of the activation we were aiming to explore. Despite this, we did find consistent activation of the SCN, particularly the left IFG, across all four social domains. This may be because, although a considerable subset of included experiments had 
high-matching control conditions, not all may have properly controlled for semantic control demands specifically. An alternative explanation is that processing socially-relevant conceptual knowledge may impose greater demands on the SCN. Consistent with this, it has been shown that processing social concepts relative to non-social concepts led to increased activation of the SCN even when controlling for potentially confounding psycholinguistic factors (Binney et al., 2016b).

\subsection{Concluding remarks and future directions}

Regions of the SCN are engaged by several types of complex social tasks, including ToM, empathy, trait inference and moral reasoning. This finding sheds light on the nature and neural correlates of the cognitive control mechanisms which contribute to the regulation of social cognition and specifically implicates processes involved in the goal-directed retrieval of conceptual knowledge. Importantly, our current findings and our broader set of hypotheses can be generalised to multiple social phenomena, thereby contributing a unified account of social cognition. Future research will need to establish a causal relationship between the SCN and successful regulation of social processing. This could be done by investigating the capacity for neurostimulation of $\mathrm{SCN}$ regions to disrupt social task performance. Similarly, whether SCN regions are sensitive to manipulations of semantic control demands within a social task could be probed directly.

Elucidating the neural bases of social control and representation may help us understand the precise nature of social impairments resulting from damage to different neural systems. For example, our framework (Binney and Ramsey, 2020) predicts that damage to representational areas such as the ATL will impair social information processing irrespective of task difficulty or the need to integrate context. In contrast, we expect that damage to control areas would lead to impaired social processing specifically when it requires selecting from amongst alternative interpretations of social cues, and/or retrieving social information that is only weakly associated with a person or a situation. Damage to perisylvian frontal and/or temporo-parietal areas (comprising the SCN) leads to semantic aphasia, a disorder characterized by impaired access and use of conceptual knowledge (Corbett et al., 2009; Jefferies et al., 2008, 2007; Jefferies and Lambon Ralph, 2006; Noonan et al., 2010). This contrasts with ATL damage which leads to semantic dementia, a condition associated with a loss or degradation of semantic knowledge (including social knowledge; Hodges and Patterson, 2007; Lambon Ralph et al., 2010; Lambon Ralph and Patterson, 2008; Patterson et al., 2007; Rogers et al., 2004). As far as we are aware, the extent to which brain damage that leads to semantic aphasia also affects social abilities has only been formally investigated in the case of emotion recognition (Souter et al., 2021). Some other insights can be found in neurodegenerative patients with prominent frontal lobe damage, where social impairments can be linked to deficits in executive function (Healey and Grossman, 2018; Kamminga et al., 2015). More generally, it will be interesting to discover whether a distinction between knowledge representation and cognitive control can inform our understanding of the precise nature of atypical or disordered social cognition in, for example, the context of dementia, acquired brain injury, autism spectrum conditions and schizophrenia.

\section{Declaration of Competing Interest}

The authors declare no potential conflicts of interest.

\section{Credit authorship contribution statement}

Veronica Diveica: Conceptualization, Methodology, Investigation, Formal analysis, Visualization, Writing - original draft, Writing - review \& editing. Kami Koldewyn: Conceptualization, Writing - review \& editing. Richard J. Binney: Conceptualization, Methodology, Writing - original draft, Writing - review \& editing, Supervision.

\section{Acknowledgements}

This research was performed as part of an all-Wales ESRC Doctoral Training Programme $+3 \mathrm{PhD}$ studentship awarded to VD [ES/P00069X/1]. The authors would like to thank Irina Giurgea for her assistance in preparing the data for publication, and Ionela Bara and Eva Balgova for their comments on a previous version of this manuscript.

\section{Data availability statement}

Following open science initiatives (Munafò et al., 2017), the current study was pre-registered via the Open Science Framework (OSF). We adhered to our pre-registered protocols (available at: osf.io/dscwv) with a few minor exceptions (see Section S1 of Supplementary Information (SI) 1 for details). All the raw datasets are openly-available on the OSF project page (available at: osf.io/fktb8/) and are accompanied by a range of study characteristics including details that are not the focus of the present study but may be of interest in future research (please see Section S1 of SI 1 for a detailed description). Moreover, the input data and output files of all analyses can be accessed via the OSF page.

A preprint of the manuscript has been deposited at www.bioRxiv.org (CC BY 4.0).

\section{Supplementary materials}

Supplementary material associated with this article can be found, in the online version, at doi:10.1016/j.neuroimage.2021.118702.

\section{References}

Ackerman, B.P., 1983. Form and function in children's understanding of ironic utterances. J. Exp. Child Psychol. 35, 487-508. doi:10.1016/0022-0965(83)90023-1.

Adolphs, R., 2010. Conceptual challenges and directions for social neuroscience. Neuron 65, 752-767. doi:10.1016/j.neuron.2010.03.006.

Adolphs, R., 2009. The social brain: neural basis of social knowledge. Annu. Rev. Psychol. 60, 693-716. doi:10.1146/annurev.psych.60.110707.163514.

Alam, G.T., Murphy, C., Smallwood, J., Jefferies, E., 2018. Meaningful inhibition: exploring the role of meaning and modality in response inhibition. Neuroimage 181, 108-119. doi:10.1016/j.neuroimage.2018.06.074.

Alcalá-López, D., Smallwood, J., Jefferies, E., Van Overwalle, F., Vogeley, K., Mars, R.B., Turetsky, B.I., Laird, A.R., Fox, P.T., Eickhoff, S.B., Bzdok, D., 2018. Computing the social brain connectome across systems and states. Cereb. Cortex 28, 2207-2232. doi:10.1093/cercor/bhx121.

Amodio, D.M., 2019. Social cognition 2.0: an interactive memory systems account. Trends Cogn. Sci. 23, 21-33. doi:10.1016/j.tics.2018.10.002.

Amodio, D.M., 2014. The neuroscience of prejudice and stereotyping. Nat. Rev. Neurosci. doi:10.1038/nrn3800.

Amodio, D.M., Cikara, M., 2021. The social neuroscience of prejudice. Annu. Rev. Psychol. 72, 439-469. doi:10.1146/annurev-psych-010419-050928.

Assem, M., Glasser, M.F., Van Essen, D.C., Duncan, J., 2020. A domain-general cognitive core defined in multimodally parcellated human cortex. Cereb. Cortex 30, 4361-4380. doi:10.1093/cercor/bhaa023.

Aviezer, H., Hassin, R.R., Ryan, J., Grady, C., Susskind, J., Anderson, A., Moscovitch, M., Bentin, S., 2008. Angry, disgusted, or afraid? Studies on the malleability of emotion perception: research article. Psychol. Sci. 19, 724-732. doi:10.1111/j.1467-9280.2008.02148.x.

Badre, D., Poldrack, R.A., Paré-Blagoev, E.J., Insler, R.Z., Wagner, A.D., 2005. Dissociable controlled retrieval and generalized selection mechanisms in ventrolateral prefrontal cortex. Neuron 47, 907-918. doi:10.1016/j.neuron.2005.07.023.

Badre, D., Wagner, A.D., 2007. Left ventrolateral prefrontal cortex and the cognitive control of memory. Neuropsychologia doi:10.1016/j.neuropsychologia.2007.06.015.

Balgova, E., Diveica, V., Walbrin, J., Binney, R.J., 2021. The ventrolateral anterior temporal lobe is commonly engaged by both mental state inference and semantic association tasks. bioRxiv 2021.09.10.459496. 10.1101/2021.09.10.459496

Barrett, L.F., Mesquita, B., Gendron, M., 2011. Context in emotion perception. Curr. Dir. Psychol. Sci. 20, 286-290. doi:10.1177/0963721411422522.

Bas-Hoogendam, J.M., van Steenbergen, H., Kreuk, T., van der Wee, N.J.A., Westenberg, P.M., 2017. How embarrassing! The behavioral and neural correlates of processing social norm violations. PLoS ONE 12, e0176326. doi:10.1371/journal.pone. 0176326.

Binder, J.R., Desai, R.H., Graves, W.W., Conant, L.L., 2009. Where is the semantic system? A critical review and meta-analysis of 120 functional neuroimaging studies. Cereb. Cortex 19, 2767-2796. doi:10.1093/cercor/bhp055.

Binney, R.J., Henry, M.L., Babiak, M., Pressman, P.S., Santos-Santos, M.A., Narvid, J. Mandelli, M.L., Strain, P.J., Miller, B.L., Rankin, K.P., Rosen, H.J., GornoTempini, M.L., 2016a. Reading words and other people: a comparison of exception word, familiar face and affect processing in the left and right temporal variants of primary progressive aphasia. Cortex 82, 147-163. doi:10.1016/j.cortex.2016.05.014. 
Binney, Richard J, Hoffman, P., Ralph, M.A.L., 2016b. Mapping the multiple graded contributions of the anterior temporal lobe representational hub to abstract and social concepts: evidence from distortion-corrected fMRI. Cereb. Cortex 26, 4227-4241. doi:10.1093/cercor/bhw260.

Binney, R.J., Parker, G.J.M., Lambon Ralph, M.A., 2012. Convergent connectivity and graded specialization in the rostral human temporal Lobe as revealed by diffusionweighted imaging probabilistic tractography. J. Cogn. Neurosci. 24, 1998-2014. doi:10.1162/jocn_a_00263.

Binney, R.J., Ramsey, R., 2020. Social semantics: the role of conceptual knowledge and cognitive control in a neurobiological model of the social brain. Neurosci. Biobehav. Rev. 112, 28-38. doi:10.1016/j.neubiorev.2020.01.030.

Bosco, F.M., Tirassa, M., Gabbatore, I., 2018. Why pragmatics and theory of mind do not (Completely) overlap. Front. Psychol. 9, 1453. doi:10.3389/fpsyg.2018.01453.

Botvinik-Nezer, R., Holzmeister, F., Camerer, C.F., Dreber, A., Huber, J., Johannesson, M., Kirchler, M., Iwanir, R., Mumford, J.A., Adcock, R.A., Avesani, P., Baczkowski, B.M., Bajracharya, A., Bakst, L., Ball, S., Barilari, M., Bault, N., Beaton, D., Beitner, J., Benoit, R.G., Berkers, R.M.W.J., Bhanji, J.P., Biswal, B.B., Bobadilla-Suarez, S., Bortolini, T., Bottenhorn, K.L., Bowring, A., Braem, S., Brooks, H.R., Brudner, E.G., Calderon, C.B., Camilleri, J.A., Castrellon, J.J., Cecchetti, L., Cieslik, E.C., Cole, Z.J., Collignon, O., Cox, R.W., Cunningham, W.A., Czoschke, S., Dadi, K., Davis, C.P., Luca, A.De, Delgado, M.R., Demetriou, L., Dennison, J.B., Di, X., Dickie, E.W., Dobryakova, E., Donnat, C.L., Dukart, J., Duncan, N.W., Durnez, J., Eed, A., Eickhoff, S.B., Erhart, A., Fontanesi, L., Fricke, G.M., Fu, S., Galván, A., Gau, R., Genon, S., Glatard, T., Glerean, E., Goeman, J.J., Golowin, S.A.E., González-García, C., Gorgolewski, K.J., Grady, C.L., Green, M.A., Guassi Moreira, J.F., Guest, O., Hakimi, S., Hamilton, J.P., Hancock, R., Handjaras, G., Harry, B.B., Hawco, C., Herholz, P., Herman, G., Heunis, S., Hoffstaedter, F., Hogeveen, J., Holmes, S., Hu, C.P., Huettel, S.A., Hughes, M.E., Iacovella, V., Iordan, A.D., Isager, P.M., Isik, A.I., Jahn, A., Johnson, M.R., Johnstone, T., Joseph, M.J.E., Juliano, A.C., Kable, J.W., Kassinopoulos, M., Koba, C., Kong, X.Z., Koscik, T.R., Kucukboyaci, N.E., Kuhl, B.A., Kupek, S., Laird, A.R., Lamm, C., Langner, R., Lauharatanahirun, N., Lee, H., Lee, S., Leemans, A., Leo, A., Lesage, E., Li, F., Li, M.Y.C., Lim, P.C., Lintz, E.N., Liphardt, S.W., Losecaat Vermeer, A.B., Love, B.C., Mack, M.L., Malpica, N., Marins, T., Maumet, C., McDonald, K., McGuire, J.T., Melero, H., Méndez Leal, A.S., Meyer, B., Meyer, K.N., Mihai, G., Mitsis, G.D., Moll, J., Nielson, D.M., Nilsonne, G., Notter, M.P., Olivetti, E., Onicas, A.I., Papale, P., Patil, K.R., Peelle, J.E., Pérez, A., Pischedda, D., Poline, J.B., Prystauka, Y., Ray, S., Reuter-Lorenz, P.A., Reynolds, R.C., Ricciardi, E., Rieck, J.R., Rodriguez-Thompson, A.M., Romyn, A., Salo, T., Samanez-Larkin, G.R., Sanz-Morales, E., Schlichting, M.L., Schultz, D.H., Shen, Q., Sheridan, M.A., Silvers, J.A., Skagerlund, K., Smith, A., Smith, D.V., Sokol-Hessner, P., Steinkamp, S.R., Tashjian, S.M., Thirion, B., Thorp, J.N., Tinghög, G., Tisdall, L., Tompson, S.H., ToroSerey, C., Torre Tresols, J.J., Tozzi, L., Truong, V., Turella, L., van 't Veer, A.E., Verguts, T., Vettel, J.M., Vijayarajah, S., Vo, K., Wall, M.B., Weeda, W.D., Weis, S., White, D.J., Wisniewski, D., Xifra-Porxas, A., Yearling, E.A., Yoon, S., Yuan, R., Yuen, K.S.L., Zhang, L., Zhang, X., Zosky, J.E., Nichols, T.E., Poldrack, R.A., Schonberg, T., 2020. Variability in the analysis of a single neuroimaging dataset by many teams. Nature 582, 84-88. doi:10.1038/s41586-020-2314-9.

Branzi, F.M., Humphreys, G.F., Hoffman, P., Lambon Ralph, M.A., 2020. Revealing the neural networks that extract conceptual gestalts from continuously evolving or changing semantic contexts. Neuroimage 220. doi:10.1016/j.neuroimage.2020.116802.

Brown, S., Schafer, E.A., 1888. An investigation into the functions of the occipital and temporal lobes of the monkey's brain. Philos. Trans. R. Soc. Lond. 179, 303-327. doi:10.1098/rstb.1888.0011.

Buckner, R.L., Andrews-Hanna, J.R., Schacter, D.L., 2008. The brain's default network: anatomy, function, and relevance to disease. Ann. N. Y. Acad. Sci. 1124, 1-38. doi:10.1196/annals.1440.011.

Button, K.S., Ioannidis, J.P.A., Mokrysz, C., Nosek, B.A., Flint, J., Robinson, E.S.J., Munafò, M.R., 2013. Power failure: why small sample size undermines the reliability of neuroscience. Nat. Rev. Neurosci. 14, 365-376. doi:10.1038/nrn3475.

Bzdok, D., Langner, R., Caspers, S., Kurth, F., Habel, U., Zilles, K., Laird, A., Eickhoff, S.B., 2011. ALE meta-analysis on facial judgments of trustworthiness and attractiveness. Brain Struct. Funct. 215, 209-223. doi:10.1007/s00429-010-0287-4.

Bzdok, D., Schilbach, L., Vogeley, K., Schneider, K., Laird, A.R., Langner, R., Eickhoff, S.B., 2012. Parsing the neural correlates of moral cognition: ALE meta-analysis on morality, theory of mind, and empathy. Brain Struct. Funct. 217, 783-796. doi:10.1007/s00429-012-0380-y.

Carp, J., 2012. On the plurality of (Methodological) worlds: estimating the analytic flexibility of fMRI experiments. Front. Neurosci. 6, 149. doi:10.3389/fnins.2012.00149.

Carruthers, P., 2017. Mindreading in adults: evaluating two-systems views. Synthese 194, 673-688. doi:10.1007/s11229-015-0792-3.

Carruthers, P., 2016. Two systems for mindreading? Rev. Philos. Psychol. 7, 141-162. doi:10.1007/s13164-015-0259-y.

Cassidy, B.S., Gutchess, A.H., 2015. Neural responses to appearance-behavior congruity. Soc. Cogn. 33, 211-226. doi:10.1521/soco.2015.33.3.1.

Castelli, F., Frith, C., Happe, F., Frith, U., 2002. Autism, Asperger syndrome and brain mechanisms for the attribution of mental states to animated shapes. Brain 125, 18391849. doi:10.1093/brain/awf189.

Chan, D., Anderson, V., Pijnenburg, Y., Whitwell, J., Barnes, J., Scahill, R., Stevens, J.M., Barkhof, F., Scheltens, P., Rossor, M.N., Fox, N.C., 2009. The clinical profile of right temporal lobe atrophy. Brain 132, 1287-1298. doi:10.1093/brain/awp037.

Chen, A.C., Welsh, R.C., Liberzon, I., Taylor, S.F., 2010. Do I like this person?” A network analysis of midline cortex during a social preference task. Neuroimage 51, 930-939. doi:10.1016/j.neuroimage.2010.02.044

Chiou, R., Humphreys, G.F., Jung, J.Y., Lambon Ralph, M.A., 2018. Controlled semantic cognition relies upon dynamic and flexible interactions between the executive 'semantic control' and hub-and-spoke 'semantic representation' systems. Cortex 103, 100-116. doi:10.1016/j.cortex.2018.02.018.

Cloutier, J., Gabrieli, J.D.E., O'Young, D., Ambady, N., 2011. An fMRI study of violations of social expectations: when people are not who we expect them to be. Neuroimage 57, 583-588. doi:10.1016/j.neuroimage.2011.04.051.

Collins, J.A., Olson, I.R., 2014. Beyond the FFA: The role of the ventral anterior temporal lobes in face processing. Neuropsychologia 61 (1), 65-79. doi:10.1016/j.neuropsychologia.2014.06.005

Corbett, F., Jefferies, E., Burns, A., Lambon Ralph, M.A., 2015. Deregulated semantic cognition contributes to object-use deficits in Alzheimer's disease: a comparison with semantic aphasia and semantic dementia. J. Neuropsychol. 9, 219-241. doi:10.1111/jnp.12047.

Corbett, F., Jefferies, E., Ehsan, S., Ralph, M.A.L., 2009. Different impairments of semantic cognition in semantic dementia and semantic aphasia: evidence from the non-verbal domain. Brain 132, 2593-2608. doi:10.1093/brain/awp146.

Cross, K.A., Torrisi, S., Reynolds Losin, E.A., Iacoboni, M., 2013. Controlling automatic imitative tendencies: interactions between mirror neuron and cognitive control systems. Neuroimage 83, 493-504. doi:10.1016/j.neuroimage.2013.06.060.

Cumming, G., 2014. The new statistics: why and how. Psychol. Sci. 25, 7-29. doi:10.1177/0956797613504966.

Cunningham, W.A., Zelazo, P.D., 2007. Attitudes and evaluations: a social cognitive neuroscience perspective. Trends Cogn. Sci. 11, 97-104. doi:10.1016/j.tics.2006.12.005.

Darda, K.M., Ramsey, R., 2019. The inhibition of automatic imitation: a meta-analysis and synthesis of fMRI studies. Neuroimage 197, 320-329. doi:10.1016/j.neuroimage.2019.04.059.

Davey, J., Cornelissen, P.L., Thompson, H.E., Sonkusare, S., Hallam, G., Smallwood, J., Jefferies, E., 2015. Automatic and controlled semantic retrieval: TMS reveals distinct contributions of posterior middle temporal gyrus and angular gyrus. J. Neurosci. 35 15230-15239. doi:10.1523/JNEUROSCI.4705-14.2015.

Davey, J., Thompson, H.E., Hallam, G., Karapanagiotidis, T., Murphy, C., De Caso, I., Krieger-Redwood, K., Bernhardt, B.C., Smallwood, J., Jefferies, E., 2016. Exploring the role of the posterior middle temporal gyrus in semantic cognition: Integration of anterior temporal lobe with executive processes. Neuroimage 137, 165-177. doi:10.1016/j.neuroimage.2016.05.051.

De Greck, M., Scheidt, L., Bölter, A.F., Frommer, J., Ulrich, C., Stockum, E., Enzi, B., Tempelmann, C., Hoffmann, T., Han, S., Northoff, G., 2012. Altered brain activity during emotional empathy in somatoform disorder. Hum. Brain Mapp. 33, 2666-2685. doi:10.1002/hbm. 21392 .

Decety, J., Lamm, C., 2007. The role of the right temporoparietal junction in social interaction: how low-level computational processes contribute to meta-cognition. Neuroscientist 13, 580-593. doi:10.1177/1073858407304654

Deen, B., Koldewyn, K., Kanwisher, N., Saxe, R., 2015. Functional organization of social perception and cognition in the superior temporal sulcus. Cereb. Cortex 25, 45964609. doi:10.1093/cercor/bhv111.

Devlin, J.T., Russell, R.P., Davis, M.H., Price, C.J., Wilson, J., Moss, H.E., Matthews, P.M., Tyler, L.K., 2000. Susceptibility-induced loss of signal: comparing PET and fMRI on a semantic task. Neuroimage 11, 589-600. doi:10.1006/nimg.2000.0595.

Diamond, A., 2013. Executive functions. Annu. Rev. Psychol. doi:10.1146/annurev-psych-113011-143750.

Ding, J., Chen, K., Liu, H., Huang, L., Chen, Y., Lv, Y., Yang, Q., Guo, Q., Han, Z., Lambon Ralph, M.A., 2020. A unified neurocognitive model of semantics language social behaviour and face recognition in semantic dementia. Nat. Commun. 11, 2595. doi:10.1038/s41467-020-16089-9.

Dobbins, I.G., Wagner, A.D., 2005. Domain-general and domain-sensitive prefrontal mechanisms for recollecting events and detecting novelty. Cereb. Cortex 15, 1768-1778. doi:10.1093/CERCOR/BHI054.

Dricu, M., Frühholz, S., 2016. Perceiving emotional expressions in others: activation likelihood estimation meta-analyses of explicit evaluation, passive perception and incidental perception of emotions. Neurosci. Biobehav. Rev. 71, 810-828. doi:10.1016/j.neubiorev.2016.10.020.

Duncan, J., 2013. The structure of cognition: attentional episodes in mind and brain. Neuron doi:10.1016/j.neuron.2013.09.015.

Duncan, J., 2010. The multiple-demand (MD) system of the primate brain: mental programs for intelligent behaviour. Trends Cogn. Sci. 14, 172-179. doi:10.1016/j.tics.2010.01.004.

Duval, C.L., Bejanin, A., Piolino, P., Laisney, M., De, V., Sayette, L., Belliard, S., Eustache, F., Atrice Desgranges, B., 2012. Theory of mind impairments in patients with semantic dementia. Brain 135, 228-241. doi:10.1093/brain/awr309.

Eickhoff, S.B., Bzdok, D., Laird, A.R., Kurth, F., Fox, P.T., 2012. Activation likelihood estimation meta-analysis revisited. Neuroimage 59, 2349-2361. doi:10.1016/j.neuroimage.2011.09.017.

Eickhoff, S.B., Bzdok, D., Laird, A.R., Roski, C., Caspers, S., Zilles, K., Fox, P.T., 2011 Co-activation patterns distinguish cortical modules, their connectivity and functional differentiation. Neuroimage 57, 938-949. doi:10.1016/j.neuroimage.2011.05.021.

Eickhoff, S.B., Laird, A.R., Fox, P.M., Lancaster, J.L., Fox, P.T., 2017. Implementation errors in the gingerALE Software: description and recommendations. Hum. Brain Mapp. 38, 7-11. doi:10.1002/hbm.23342.

Eickhoff, S.B., Laird, A.R., Grefkes, C., Wang, L.E., Zilles, K., Fox, P.T., 2009. Coordinatebased activation likelihood estimation meta-analysis of neuroimaging data: a randomeffects approach based on empirical estimates of spatial uncertainty. Hum. Brain Mapp. 30, 2907-2926. doi:10.1002/hbm.20718.

Eickhoff, S.B., Nichols, T.E., Laird, A.R., Hoffstaedter, F., Amunts, K., Fox, P.T., Bzdok, D., Eickhoff, C.R., 2016. Behavior, sensitivity, and power of activation likelihood estimation characterized by massive empirical simulation. Neuroimage $137,70-85$. doi:10.1016/j.neuroimage.2016.04.072. 
Embleton, K.V., Haroon, H.A., Morris, D.M., Ralph, M.A.L., Parker, G.J.M., 2010. Distortion correction for diffusion-weighted MRI tractography and fMRI in the temporal lobes. Hum. Brain Mapp. 31, 1570-1587. doi:10.1002/hbm.20959.

Engell, A.D., Haxby, J.V., Todorov, A., 2007. Implicit trustworthiness decisions: automatic coding of face properties in the human amygdala. J. Cogn. Neurosci. 19, 1508-1519. doi:10.1162/jocn.2007.19.9.1508.

Eres, R., Louis, W.R., Molenberghs, P., 2018. Common and distinct neural networks involved in fMRI studies investigating morality: an ALE meta-analysis. Soc. Neurosci. 13, 384-398. doi:10.1080/17470919.2017.1357657.

Fan, Y., Duncan, N.W., de Greck, M., Northoff, G., 2011. Is there a core neural network in empathy? An fMRI based quantitative meta-analysis. Neurosci. Biobehav. Rev. 35, 903-911. doi:10.1016/j.neubiorev.2010.10.009.

Fedorenko, E., Duncan, J., Kanwisher, N., 2013. Broad domain generality in focal regions of frontal and parietal cortex. Proc. Natl. Acad. Sci. U. S. A. 110, 16616-16621. doi:10.1073/pnas.1315235110.

Ferguson, M.J., Mann, T.C., Wojnowicz, M.T., 2014. Rethinking duality: criticisms and ways forward. In: Sherman, J.W., Gawronski, B., Trope, Y. (Eds.), Dual-Process Theories of the Social Mind. The Guilford Press, New York, pp. 578-594.

Fidler, K., Hütter, M., 2014. The limits of automaticity. In: Sherman, J.W., Gawronski, B., Trope, Y. (Eds.), Dual-Process Theories of the Social Mind. The Guilford Press, New York, pp. 497-513.

Forbes, C.E., Grafman, J., 2013. Social neuroscience: the second phase. Front. Hum. Neurosci. 7, 20. doi:10.3389/fnhum.2013.00020.

Freeman, J.B., Johnson, K.L., 2016. More Than Meets the Eye: split-Second Social Perception. Trends Cogn. Sci. 20, 362-374. doi:10.1016/j.tics.2016.03.003.

Frith, C.D., Frith, U., 2006. The Neural Basis of Mentalizing. Neuron 50 (4), 531-534. doi:10.1016/j.neuron.2006.05.001.

Frith, C.D., Frith, U., 2012. Mechanisms of Social Cognition. Annu. Rev. Psychol 63, 287313. doi:10.1146/annurev-psych-120710-100449.

Frith, C.D., 2007. The social brain? Philosophical Transactions of the Royal Society B: Biological Sciences 362 (1480), 671-678. doi:10.1098/RSTB.2006.2003.

Frith, U., Frith, C.D., 2003. Development and neurophysiology of mentalizing. Philos. Trans. R. Soc. London. Ser. B Biol. Sci. 358, 459-473. doi:10.1098/rstb.2002.1218.

Fujita, K., Trope, Y., Cunningham, W.A., Liberman, N., 2014. What is control? A conceptual analysis. In: Sherman, J., Gawronski, B., Trope, Y. (Eds.), Dual-Process Theories of the Social Mind. Guilford Press, New York, pp. 50-68.

Gao, Z., Zheng, L., Chiou, R., Gouws, A., Krieger-Redwood, K., Wang, X., Varga, D., Ralph, M.A.L., Smallwood, J., Jefferies, E., 2020. Distinct and common neural coding of semantic and non-semantic control demands. bioRxiv. 10.1101/2020.11.16.384883

Gefen, T., Wieneke, C., Martersteck, A., Whitney, K., Weintraub, S., Mesulam, M.M., Rogalski, E., 2013. Naming vs knowing faces in primary progressive aphasia: a tale of 2 hemispheres. Neurology 81, 658-664. doi:10.1212/WNL.0b013e3182a08f83.

Gilbert, S.J., Burgess, P.W., 2008. Executive function. Curr. Biol. 18, R110-R114. doi:10.1016/j.cub.2007.12.014.

Gold, B.T., Balota, D.A., Jones, S.J., Powell, D.K., Smith, C.D., Andersen, A.H., 2006. Dissociation of automatic and strategic lexical-semantics: functional magnetic resonance imaging evidence for differing roles of multiple frontotemporal regions. J. Neurosci. 26, 6523-6532. doi:10.1523/JNEUROSCI.0808-06.2006.

Greven, I.M., Downing, P.E., Ramsey, R., 2016. Linking person perception and person knowledge in the human brain. Soc. Cogn. Affect. Neurosci. 11, 641-651. doi:10.1093/scan/nsv148.

Hall, J., Philip, R.C.M., Marwick, K., Whalley, H.C., Romaniuk, L., McIntosh, A.M., Santos, I., Sprengelmeyer, R., Johnstone, E.C., Stanfield, A.C., Young, A.W., Lawrie, S.M., 2012. Social cognition, the male brain and the autism spectrum. PLoS ONE 7, e49033. doi:10.1371/journal.pone.0049033.

Happé, F., Cook, J.L., Bird, G., 2017. The structure of social cognition: in(Ter)dependence of sociocognitive processes. Annu. Rev. Psychol. doi:10.1146/annurev-psych-010416-044046.

Hassabis, D., Spreng, R.N., Rusu, A.A., Robbins, C.A., Mar, R.A., Schacter, D.L., 2014. Imagine all the people: how the brain creates and uses personality models to predict behavior. Cereb. Cortex 24, 1979-1987. doi:10.1093/cercor/bht042.

Healey, M.L., Grossman, M., 2018. Cognitive and affective perspective-taking: evidence for shared and dissociable anatomical substrates. Front. Neurol. 9, 491. doi:10.3389/fneur.2018.00491.

Hehman, E., Ingbretsen, Z.A., Freeman, J.B., 2014. The neural basis of stereotypic impact on multiple social categorization. Neuroimage 101, 704-711. doi:10.1016/j.neuroimage.2014.07.056

Henson, R., 2006. Forward inference using functional neuroimaging: dissociations versus associations. Trends Cogn. Sci. 10, 64-69. doi:10.1016/j.tics.2005.12.005.

Heyes, C., 2014. Submentalizing. Perspect. Psychol. Sci. 9, 131-143. doi:10.1177/1745691613518076.

Hodges, J.R., Patterson, K., 2007. Semantic dementia: a unique clinicopathological syndrome. Lancet Neurol. 6, 1004-1014. doi:10.1016/S1474-4422(07)70266-1.

Hodgson, C., Lambon Ralph, M.A., 2008. Mimicking aphasic semantic errors in normal speech production: evidence from a novel experimental paradigm. Brain Lang 104, 89-101. doi:10.1016/J.BANDL.2007.03.007.

Hodgson, V.J., Lambon Ralph, M.A., Jackson, R.L., 2021. Multiple dimensions underlying the functional organization of the language network. Neuroimage 241, 118444. doi:10.1016/J.NEUROIMAGE.2021.118444.

Huang, Y., Su, L., Ma, Q., 2020. The stroop effect: an activation likelihood estimation meta-analysis in healthy young adults. Neurosci. Lett. 716, 134683. doi:10.1016/j.neulet.2019.134683.

Huey, E.D., Krueger, F., Grafman, J., 2006. Representations in the human prefrontal cortex. Curr. Dir. Psychol. Sci. 15, 167-171. doi:10.1111/j.1467-8721.2006.00429.x.

Hugdahl, K., Raichle, M.E., Mitra, A., Specht, K., 2015. On the existence of a generalized non-specific task-dependent network. Front. Hum. Neurosci. 9, 1-15. doi:10.3389/fnhum.2015.00430.

Humphreys, G., Ralph, M.L., Simons, J., 2020. A Unifying account of angular gyrus contributions to episodic and semantic cognition. PsyArXiv. 10.31234/OSF.IO/R2DEU

Humphreys, G.F., Hoffman, P., Visser, M., Binney, R.J., Lambon Ralph, M.A., 2015. Establishing task- and modality-dependent dissociations between the semantic and default mode networks. Proc. Natl. Acad. Sci. U. S. A. 112, 7857-7862. doi:10.1073/pnas.1422760112.

Humphreys, G.F., Lambon Ralph, M.A., 2015a. Fusion and fission of cognitive functions in the human parietal cortex. Cereb. Cortex 25, 3547-3560. doi:10.1093/cercor/bhu198.

Humphreys, G.F., Lambon Ralph, M.A., 2015b. Fusion and fission of cognitive functions in the human parietal cortex. Cereb. Cortex 25, 3547-3560. doi:10.1093/cercor/bhu198.

Humphreys, G., Lambon Ralph, M.A., 2017. Mapping domain-selective and counterpointed domain-general higher cognitive functions in the lateral parietal cortex: evidence from fMRI comparisons of difficulty. Cereb. Cortex 27, 1499-4212. doi:10.1093/cercor/bhx107.

Igelström, K.M., Graziano, M.S.A., 2017. The inferior parietal lobule and temporoparietal junction: a network perspective. Neuropsychologia 105, 70-83. doi:10.1016/j.neuropsychologia.2017.01.001.

Irish, M., Hodges, J.R., Piguet, O., 2014. Right anterior temporal lobe dysfunction underlies theory of mind impairments in semantic dementia. Brain 137, 1241-1253. doi:10.1093/brain/awu003.

Jackson, R.L., 2021. The neural correlates of semantic control revisited. Neuroimage 224, 117444. doi:10.1016/j.neuroimage.2020.117444

Jackson, R.L., Cloutman, L.L., Lambon Ralph, M.A., 2019. Exploring distinct default mode and semantic networks using a systematic ICA approach. Cortex 113, 279-297. doi:10.1016/j.cortex.2018.12.019.

Jackson, R.L., Humphreys, G.F., Rice, G.E., Binney, R.J., Lambon Ralph, M.A., 2021. The coherent default mode network is not involved in episodic recall or social cognition. bioRxiv doi:10.1101/2021.01.08.425921.

January, D., Trueswell, J.C., Thompson-Schill, S.L., 2009. Co-localization of stroop and syntactic ambiguity resolution in Broca's area: implications for the neural basis of sentence processing. J. Cogn. Neurosci. 21, 2434-2444. doi:10.1162/jocn.2008.21179.

Jefferies, E., 2013. The neural basis of semantic cognition: converging evidence from neuropsychology, neuroimaging and TMS. Cortex 49, 611-625. doi:10.1016/j.cortex.2012.10.008.

Jefferies, E., Baker, S.S., Doran, M., Ralph, M.A.L., 2007. Refractory effects in stroke aphasia: a consequence of poor semantic control. Neuropsychologia 45, 1065-1079. doi:10.1016/j.neuropsychologia.2006.09.009.

Jefferies, E., Lambon Ralph, M.A., 2006. Semantic impairment in stroke aphasia versus semantic dementia: a case-series comparison. Brain 129, 2132-2147. doi:10.1093/brain/awl153.

Jefferies, E., Patterson, K., Ralph, M.A.L., 2008. Deficits of knowledge versus executive control in semantic cognition: insights from cued naming. Neuropsychologia 46, 649 658. doi:10.1016/j.neuropsychologia.2007.09.007.

Jezzard, P., Clare, S., 1999. Sources of distortion in functional MRI data. In: Human Brain Mapping. John Wiley \& Sons, Ltd, pp. 80-85. doi:10.1002/(SICI)1097-0193(1999)8:2/3-80::AID-HBM2-3.0.CO;2-C.

Kamminga, J., Kumfor, F., Burrell, J.R., Piguet, O., Hodges, J.R., Irish, M., 2015. Differentiating between right-lateralised semantic dementia and behavioural-variant frontotemporal dementia: an examination of clinical characteristics and emotion processing. $\mathrm{J}$. Neurol. Neurosurg. Psychiatry 86 (1082), LP-1088. doi:10.1136/jnnp-2014-309120.

Klüver, Heinrich, Bucy, P.C., 1939. Preliminary analysis of functions of the temporal lobes in monkeys. Arch. Neurol. Psychiatry 42, 979-1000. doi:10.1001/archneurpsyc.1939.02270240017001.

Korman, J., Malle, B.F., 2016. Grasping for traits or reasons? How people grapple with puzzling social behaviors. Personal. Soc. Psychol. Bull. 42, 1451-1465. doi:10.1177/0146167216663704.

Krieger-Redwood, K., Teige, C., Davey, J., Hymers, M., Jefferies, E., 2015. Conceptual control across modalities: graded specialisation for pictures and words in inferior frontal and posterior temporal cortex. Neuropsychologia 76, 92-107. doi:10.1016/j.neuropsychologia.2015.02.030

Lambon Ralph, M.A., Jefferies, E., Patterson, K., Rogers, T.T., 2017. The neural and computational bases of semantic cognition. Nat. Rev. Neurosci. 18, 42-55. doi:10.1038/nrn.2016.150

Lambon Ralph, M.A., Patterson, K., 2008. Generalization and differentiation in semantic memory insights from semantic dementia. Ann. N. Y. Acad. Sci. 1124, 61-76. doi:10.1196/annals.1440.006.

Lambon Ralph, M.A., Sage, K., Jones, R.W., Mayberry, E.J., 2010. Coherent concepts are computed in the anterior temporal lobes. Proc. Natl. Acad. Sci. U. S. A. 107, 27172722. doi: $10.1073 /$ pnas.0907307107.

Lancaster, J.L., Tordesillas-Gutiérrez, D., Martinez, M., Salinas, F., Evans, A., Zilles, K., Mazziotta, J.C., Fox, P.T., 2007. Bias between MNI and talairach coordinates analyzed using the ICBM-152 brain template. Hum. Brain Mapp. 28, 1194-1205. doi:10.1002/hbm.20345.

Lavoie, M.A., Vistoli, D., Sutliff, S., Jackson, P.L., Achim, A.M., 2016. Social representations and contextual adjustments as two distinct components of the Theory of Mind brain network: evidence from the REMICS task. Cortex 81, 176-191. doi:10.1016/j.cortex.2016.04.017.

Lieberman, M.D., 2007. Social cognitive neuroscience: a review of core processes. Annu. Rev. Psychol. 58, 259-289. doi:10.1146/annurev.psych.58.110405.085654.

Lindquist, K.A., Gendron, M., Barrett, L.F., Dickerson, B.C., 2014. Emotion perception, but not affect perception, is impaired with semantic memory loss. Emotion 14, 375-387. doi:10.1037/a0035293 
Lindquist, K.A, Wager, T.D., Kober, H., Bliss-Moreau, E., Barrett, L.F., 2012. The brain basis of emotion: A meta-analytic review. Behavioral and Brain Sciences 35 (3), 121143. doi:10.1017/S0140525X11000446.

Ma, N., Vandekerckhove, M., Baetens, K., Overwalle, F.Van, Seurinck, R., Fias, W., 2012. Inconsistencies in spontaneous and intentional trait inferences. Soc. Cogn. Affect. Neurosci. 7, 937-950. doi:10.1093/scan/nsr064.

Malle, B., Holbrook, J., 2012. Is There a Hierarchy of Social Inferences? The likelihood and speed of inferring intentionality, mind, and personality. Artic. J. Personal. Soc. Psychol. 102, 661-684. doi:10.1037/a0026790.

Margulies, D.S., Ghosh, S.S., Goulas, A., Falkiewicz, M., Huntenburg, J.M., Langs, G., Bezgin, G., Eickhoff, S.B., Castellanos, F.X., Petrides, M., Jefferies, E., Smallwood, J., 2016. Situating the default-mode network along a principal gradient of macroscale cortical organization. Proc. Natl. Acad. Sci. U. S. A. 113, 12574-12579. doi:10.1073/pnas.1608282113.

Mars, R.B., Neubert, F.X., Noonan, M.P., Sallet, J., Toni, I., Rushworth, M.F.S., 2012. On the relationship between the "default mode network" and the "social brain". Front. Hum. Neurosci. 6, 1-9. doi:10.3389/fnhum.2012.00189.

Mason, R.A., Williams, D.L., Kana, R.K., Minshew, N., Just, M.A., 2008. Theory of Mind disruption and recruitment of the right hemisphere during narrative comprehension in autism. Neuropsychologia 46, 269-280. doi:10.1016/j.neuropsychologia.2007.07.018.

Melnikoff, D.E., Bargh, J.A., 2018. The mythical number two. Trends Cogn. Sci. doi:10.1016/j.tics.2018.02.001.

Mende-Siedlecki, P., Baron, S.G., Todorov, A., 2013a. Diagnostic value underlies asymmetric updating of impressions in the morality and ability domains. J. Neurosci. 33, 19406-19415. doi:10.1523/JNEUROSCI.2334-13.2013.

Mende-Siedlecki, P., Cai, Y., Todorov, A., 2013b. The neural dynamics of updating person impressions. Soc. Cogn. Affect. Neurosci. 8, 623-631. doi:10.1093/scan/nss040.

Mende-Siedlecki, P., Said, C.P., Todorov, A., 2013. The social evaluation of faces: a metaanalysis of functional neuroimaging studies. Soc. Cogn. Affect. Neurosci. 8, 285-299. doi:10.1093/scan/nsr090.

Mende-Siedlecki, P., Todorov, A., 2016. Neural dissociations between meaningful and mere inconsistency in impression updating. Soc. Cogn. Affect. Neurosci. 11, 14891500. doi:10.1093/scan/nsw058.

Meyer, M.L., Spunt, R.P., Berkman, E.T., Taylor, S.E., Lieberman, M.D., 2012. Evidence for social working memory from a parametric functional MRI study. PNAS 109, 18831888. doi:10.1073/pnas.1121077109.

Mier, D., Sauer, C., Lis, S., Esslinger, C., Wilhelm, J., Gallhofer, B., Kirsch, P., 2010. Neuronal correlates of affective theory of mind in schizophrenia out-patients: evidence for a baseline deficit. Psychol. Med. 40, 1607-1617. doi:10.1017/S0033291709992133.

Mitchell, R.L.C., 2013. Further characterisation of the functional neuroanatomy associated with prosodic emotion decoding. Cortex 49, 1722-1732. doi:10.1016/j.cortex.2012.07.010.

Molenberghs, P., Johnson, H., Henry, J.D., Mattingley, J.B., 2016. Understanding the minds of others: a neuroimaging meta-analysis. Neurosci. Biobehav. Rev. 65, 276291. doi:10.1016/j.neubiorev.2016.03.020.

Moll, J., Zahn, R., De Oliveira-Souza, R., Krueger, F., Grafman, J., 2005. The neural basis of human moral cognition. Nature Reviews Neuroscience 6 (10), 799-809. doi: $10.1038 / \mathrm{nrn} 1768$.

Müller, V.I., Cieslik, E.C., Laird, A.R., Fox, P.T., Radua, J., Mataix-Cols, D., Tench, C.R., Yarkoni, T., Nichols, T.E., Turkeltaub, P.E., Wager, T.D., Eickhoff, S.B., 2018. Ten simple rules for neuroimaging meta-analysis. Neurosci. Biobehav. Rev. 84, 151-161. doi:10.1016/j.neubiorev.2017.11.012.

Munafò, M.R., Nosek, B.A., Bishop, D.V.M., Button, K.S., Chambers, C.D., Percie Du Sert, N., Simonsohn, U., Wagenmakers, E.J., Ware, J.J., Ioannidis, J.P.A., 2017. A manifesto for reproducible science. Nat. Hum. Behav, doi:10.1038/s41562-016-0021.

Nagel, I.E., Schumacher, E.H., Goebel, R., D'Esposito, M., 2008. Functional MRI investigation of verbal selection mechanisms in lateral prefrontal cortex. Neuroimage 43, 801-807. doi:10.1016/j.neuroimage.2008.07.017.

Nee, D.E., Wager, T.D., Jonides, J., 2007. Interference resolution: insights from a meta-analysis of neuroimaging tasks. Cogn. Affect. Behav. Neurosci. 7, 1-17. doi:10.3758/CABN.7.1.1.

Nichols, T., Brett, M., Andersson, J., Wager, T., Poline, J.B., 2005. Valid conjunction inference with the minimum statistic. Neuroimage 25, 653-660. doi:10.1016/j.neuroimage.2004.12.005

Noonan, K.A., Jefferies, E., Corbett, F., Lambon Ralph, M.A., 2010. Elucidating the nature of deregulated semantic cognition in semantic aphasia: evidence for the roles of prefrontal and temporo-parietal cortices. J. Cogn. Neurosci. 22, 1597-1613. doi:10.1162/jocn.2009.21289.

Noonan, K.A., Jefferies, E., Visser, M., Lambon Ralph, M.A., 2013. Going beyond inferior prefrontal involvement in semantic control: evidence for the additional contribution of dorsal angular gyrus and posterior middle temporal cortex. J. Cogn. Neurosci. 25, 1824-1850. doi:10.1162/jocn a 00442.

Numssen, O., Bzdok, D., Hartwigsen, G., 2021. Functional specialization within the inferior parietal lobes across cognitive domains. Elife 10. doi:10.7554/eLife.63591.

Ojemann, J.G., Akbudak, E., Snyder, A.Z., McKinstry, R.C., Raichle, M.E., Conturo, T.E., 1997. Anatomic localization and quantitative analysis of gradient refocused echo-planar fMRI susceptibility artifacts. Neuroimage 6, 156-167. doi:10.1006/nimg.1997.0289.

Olson, I.R., Mccoy, D., Klobusicky, E., Ross, L.A., 2013. Social cognition and the anterior temporal lobes: a review and theoretical framework. Soc. Cogn. Affect. Neurosci. 8, 123-133. doi:10.1093/scan/nss119.

Özdem, C., Brass, M., Van der Cruyssen, L., Van Overwalle, F., 2017. The overlap between false belief and spatial reorientation in the temporo-parietal junction: the role of input modality and task. Soc. Neurosci. 12, 207-217. doi:10.1080/17470919.2016.1143027.

Patterson, K., Nestor, P.J., Rogers, T.T., 2007. Where do you know what you know?
The representation of semantic knowledge in the human brain. Nat. Rev. Neurosci. doi:10.1038/nrn2277.

Perry, R.J., Rosen, H.R., Kramer, J.H., Beer, J.S., Levenson, R.L., Miller, B.L., 2001 Hemispheric dominance for emotions, empathy and social behaviour: evidence from right and left handers with frontotemporal dementia. Neurocase 7, 145-160. doi:10.1093/NEUCAS/7.2.145.

Petersen, S.E., Posner, M.I., 2012. The attention system of the human brain: 20 years after. Annu. Rev. Neurosci. 35, 73-89. doi:10.1146/annurev-neuro-062111-150525.

Pexman, P.M., 2008. It's fascinating research: the cognition of verbal irony. Curr. Dir. Psychol. Sci. 17, 286-290. doi:10.1111/j.1467-8721.2008.00591.x.

Plaut, D.C., 2002. Graded modality-specific specialisation in semantics: a computational account of optic aphasia. Cogn. Neuropsychol. 19, 603-639. doi:10.1080/02643290244000112.

Quadflieg, S., Koldewyn, K., 2017. The neuroscience of people watching: how the human brain makes sense of other people's encounters. Ann. N. Y. Acad. Sci. 1396, 166-182. doi:10.1111/nyas.13331.

Quesque, F., Rossetti, Y., 2020. What do theory-of-mind tasks actually measure? Theory and practice. Perspect. Psychol. Sci. 15, 384-396. doi:10.1177/1745691619896607.

Quinn, K.A., Rosenthal, H.E.S., 2012. Categorizing others and the self: how social memory structures guide social perception and behavior. Learn. Motiv. 43, 247-258. doi:10.1016/j.lmot.2012.05.008.

Ramanan, S., Bellana, B., 2019. A domain-general role for the angular gyrus in retrieving internal representations of the external world. J. Neurosci. 39, 2978-2980. doi:10.1523/JNEUROSCI.3231-18.2019.

Ramanan, S., Piguet, O., Irish, M., 2018. Rethinking the role of the angular gyrus in remembering the past and imagining the future: the contextual integration model. Neuroscientist doi: $10.1177 / 1073858417735514$.

Ramsey, R., Ward, R., 2020. Putting the nonsocial into social neuroscience: a role for domain-general priority maps during social interactions. Perspect. Psychol. Sci. doi:10.1177/1745691620904972.

Rankin, K.P., Kramer, J.H., Miller, B.L., 2005. Patterns of cognitive and emotional empathy in frontotemporal lobar degeneration. Cogn. Behav. Neurol. 18, 28-36. doi:10.1097/01.wnn.0000152225.05377.ab.

Reniers, R.L.E.P., Corcoran, R., Völlm, B.A., Mashru, A., Howard, R., Liddle, P.F., 2012 Moral decision-making, ToM, empathy and the default mode network. Biol. Psychol. 90, 202-210. doi:10.1016/j.biopsycho.2012.03.009.

Rice, G.E., Hoffman, P., Binney, R.J., Lambon Ralph, M.A., 2018. Concrete versus abstract forms of social concept: an fMRI comparison of knowledge about people versus social terms. Philos. Trans. R. Soc. B Biol. Sci. 373, 20170136. doi:10.1098/rstb.2017.0136.

Rice, G.E., Hoffman, P., Lambon Ralph, M.A., 2015. Graded specialization within and between the anterior temporal lobes. Ann. N. Y. Acad. Sci. 1359, 84-97. doi:10.1111/nyas.12951.

Rilling, J.K., Dagenais, J.E., Goldsmith, D.R., Glenn, A.L., Pagnoni, G., 2008. Social cognitive neural networks during in-group and out-group interactions. Neuroimage 41 , 1447-1461. doi:10.1016/j.neuroimage.2008.03.044.

Rodd, J.M., 2020. Settling into semantic space: an ambiguity-focused account of word-meaning access. Perspect. Psychol. Sci. 15, 411-427. doi:10.1177/1745691619885860.

Rogers, T.T., Lambon Ralph, M.A., Garrard, P., Bozeat, S., McClelland, J.L., Hodges, J.R., Patterson, K., 2004. Structure and deterioration of semantic memory: a neuropsychological and computational investigation. Psychol. Rev. 111, 205-235. doi:10.1037/0033-295X.111.1.205.

Rosen, H.J., Pace-Savitsky, K., Perry, R.J., Kramer, J.H., Miller, B.L., Levenson, R.W., 2004 Recognition of emotion in the frontal and temporal variants of frontotemporal dementia. Dement. Geriatr. Cogn. Disord. 17, 277-281. doi:10.1159/000077154.

Rothmayr, C., Sodian, B., Hajak, G., Döhnel, K., Meinhardt, J., Sommer, M., 2011. Common and distinct neural networks for false-belief reasoning and inhibitory control. Neuroimage 56, 1705-1713. doi:10.1016/j.neuroimage.2010.12.052.

RStudio Team, 2020. RStudio: integrated development for R [WWW Document]. URL https://www.rstudio.com/ (accessed 3.17.21).

Samson, D., Apperly, I.A., Kathirgamanathan, U., Humphreys, G.W., 2005. Seeing it my way: a case of a selective deficit in inhibiting self-perspective. Brain 128, 1102-1111. doi:10.1093/brain/awh464

Satpute, A.B., Badre, D., Ochsner, K.N., 2014. Distinct regions of prefrontal cortex are associated with the controlled retrieval and selection of social information. Cereb. Cortex 24, 1269-1277. doi:10.1093/cercor/bhs408.

Satpute, A.B., Lieberman, M.D., 2006. Integrating automatic and controlled processes into neurocognitive models of social cognition. 10.1016/j.brainres.2006.01.005

Saxe, R., Wexler, A., 2005. Making sense of another mind: the role of the right temporo-parietal junction. Neuropsychologia 43, 1391-1399. doi:10.1016/j.neuropsychologia.2005.02.013.

Schurz, M., Aichhorn, M., Martin, A., Perner, J., 2013. Common brain areas engaged in false belief reasoning and visual perspective taking: a meta-analysis of functional brain imaging studies. Front. Hum. Neurosci. 7, 712. doi:10.3389/fnhum.2013.00712.

Schurz, M., Radua, J., Aichhorn, M., Richlan, F., Perner, J., 2014. Fractionating theory of mind: a meta-analysis of functional brain imaging studies. Neurosci. Biobehav. Rev. 42, 9-34. doi:10.1016/j.neubiorev.2014.01.009.

Schurz, M., Radua, J., Tholen, M.G., Maliske, L., Margulies, D.S., Mars, R.B., Sallet, J., Kanske, P., 2020. Toward a hierarchical model of social cognition: a neuroimaging meta-analysis and integrative review of empathy and theory of mind. Psychol. Bull. doi: $10.1037 /$ bul0000303, undefined, undefined.

Schurz, M., Tholen, M.G., Perner, J., Mars, R.B., Sallet, J., 2017. Specifying the brain anatomy underlying temporo-parietal junction activations for theory of mind: a review using probabilistic atlases from different imaging modalities. Hum. Brain Mapp. doi:10.1002/hbm.23675 
Sherman, J.W., Krieglmeyer, R., Calanchini, J., 2014. Process models require process measures. In: Sherman, J., Gawronski, B., Trope, Y. (Eds.), Dual-Process Theories of the Social Mind. Guilford Press, New York, pp. 121-138.

Shiffrin, R.M., Schneider, W., 1977. Controlled and automatic human information processing: II. Perceptual learning, automatic attending and a general theory. Psychol. Rev. 84, 127-190. doi:10.1037/0033-295X.84.2.127.

Smallwood, J., Tipper, C., Brown, K., Baird, B., Engen, H., Michaels, J.R., Grafton, S., Schooler, J.W., 2013. Escaping the here and now: evidence for a role of the default mode network in perceptually decoupled thought. Neuroimage 69, 120-125. doi:10.1016/j.neuroimage.2012.12.012.

Snowden, J.S., Thompson, J.C., Neary, D., 2012. Famous people knowledge and the right and left temporal lobes. In: Behavioural Neurology. Hindawi Limited, pp. 35-44. doi:10.3233/BEN-2012-0347.

Snowden, J.S., Thompson, J.C., Neary, D., Snowden, Julie S., 2004. Knowledge of famous faces and names in semantic dementia. Brain 127, 860-872. doi:10.1093/brain/awh099.

Soch, J., Deserno, L., Assmann, A., Barman, A, Walter, H., Richardson-Klavehn, A., Schott, B.H., 2017. Inhibition of information flow to the default mode network during self-reference versus reference to others. Cereb. Cortex 27, 3930-3942. doi:10.1093/cercor/bhw206.

Souter, N.E., Lindquist, K., Jefferies, B., 2021. Impaired emotion perception and categorization in semantic aphasia. PsyArXiV. 10.31234/OSF.IO/CY37Z

Spreng, R.N., Andrews-Hanna, J.R., 2015. The default network and social cognition. In: Toga, A.W. (Ed.), Brain Mapping: An Encyclopedic Reference. Academic press, Oxford, UK, pp. 165-169. doi:10.1016/B978-0-12-397025-1.00173-1.

Tamm, S., Nilsonne, G., Schwarz, J., Lamm, C., Kecklund, G., Petrovic, P., Fischer, H., Åkerstedt, T., Lekander, M., 2017. The effect of sleep restriction on empathy for pain: an fMRI study in younger and older adults. Sci. Rep. 7, 1-14. doi:10.1038/s41598-017-12098-9.

Thompson-Schill, S.L., D’Esposito, M., Aguirre, G.K., Farah, M.J., 1997. Role of left inferior prefrontal cortex in retrieval of semantic knowledge: a reevaluation. Proc. Natl. Acad. Sci. U. S. A. 94, 14792-14797. doi:10.1073/pnas.94.26.14792.

Thompson, H.E., Almaghyuli, A., Noonan, K.A., Barak, O., Lambon Ralph, M.A., Jefferies, E., 2018. The contribution of executive control to semantic cognition: convergent evidence from semantic aphasia and executive dysfunction. J. Neuropsychol. 12, 312-340. doi:10.1111/jnp.12142.

Thompson, S.A., Patterson, K., Hodges, J.R., 2003. Left/right asymmetry of atrophy in semantic dementia. Neurology 61 (1196), LP-1203. doi:10.1212/01.WNL.0000091868.28557.B8.

Timmers, I., Park, A.L., Fischer, M.D., Kronman, C.A., Heathcote, L.C., Hernandez, J.M., Simons, L.E., 2018. Is empathy for pain unique in its neural correlates? A meta-analysis of neuroimaging studies of empathy. Front. Behav. Neurosci. 12, 289. doi:10.3389/fnbeh.2018.00289.
Turkeltaub, P.E., Eickhoff, S.B., Laird, A.R., Fox, M., Wiener, M., Fox, P., 2012. Minimizing within-experiment and within-group effects in activation likelihood estimation metaanalyses. Hum. Brain Mapp. 33, 1-13. doi:10.1002/hbm.21186.

Uleman, J.S., Saribay, S.A., Gonzalez, C.M., 2007. Spontaneous inferences, implicit impressions, and implicit theories. Annu. Rev. Psychol. 59, 329-360. doi:10.1146/annurev.psych.59.103006.093707.

Van der Meer, L., Groenewold, N.A., Nolen, W.A., Pijnenborg, M., Aleman, A., 2011. Inhibit yourself and understand the other: neural basis of distinct processes underlying Theory of Mind. Neuroimage 56, 2364-2374. doi:10.1016/j.neuroimage.2011.03.053.

Van Overwalle, F., 2009. Social cognition and the brain: a meta-analysis. Hum. Brain Mapp doi:10.1002/hbm.20547.

Van Overwalle, F., Vandekerckhove, M., 2013. Implicit and explicit social mentalizing: dual processes driven by a shared neural network. Front. Hum. Neurosci. 7. doi:10.3389/fnhum.2013.00560.

Visser, M., Jefferies, E., Lambon Ralph, M.A., 2010. Semantic processing in the anterior temporal lobes: a meta-analysis of the functional neuroimaging literature. J. Cogn. Neurosci. 22, 1083-1094. doi:10.1162/jocn.2009.21309.

Wagner, A.D., Paré-Blagoev, E.J., Clark, J., Poldrack, R.A., 2001. Recovering meaning: left prefrontal cortex guides controlled semantic retrieval. Neuron 31, 329-338. doi:10.1016/S0896-6273(01)00359-2.

Wang, A.T., Lee, S.S., Sigman, M., Dapretto, M., 2006. Developmental changes in the neural basis of interpreting communicative intent. Soc. Cogn. Affect. Neurosci. 1, 107121. doi:10.1093/scan/ns1018.

Wang, X., Bernhardt, B.C., Karapanagiotidis, T., De Caso, I., Gonzalez Alam, T.R.del J., Cotter, Z., Smallwood, J., Jefferies, E., 2018. The structural basis of semantic control: evidence from individual differences in cortical thickness. Neuroimage 181, 480-489. doi:10.1016/J.NEUROIMAGE.2018.07.044

Wang, X., Margulies, D.S., Smallwood, J., Jefferies, E., 2020. A gradient from long-term memory to novel cognition: transitions through default mode and executive cortex. Neuroimage 220. doi:10.1101/2020.01.16.908327.

Weissman, D.H., Perkins, A.S., Woldorff, M.G., 2008. Cognitive control in social situations: a role for the dorsolateral prefrontal cortex. Neuroimage 40, 955-962. doi:10.1016/j.neuroimage.2007.12.021.

Whitney, C., Kirk, M., O'Sullivan, J., Ralph, M.A.L., Jefferies, E., 2012. Executive semantic processing is underpinned by a large-scale neural network: revealing the contribution of left prefrontal, posterior temporal, and parietal cortex to controlled retrieval and selection using TMS. J. Cogn. Neurosci. 24, 133-147. doi:10.1162/jocn a 00123.

Zahn, R., Moll, J., Krueger, F., Huey, E.D., Garrido, G., Grafman, J., 2007. Social concepts are represented in the superior anterior temporal cortex. Proc. Natl. Acad. Sci. U. S. A. 104, 6430-6435. doi:10.1073/pnas.0607061104.

Zaki, J., Hennigan, K., Weber, J., Ochsner, K.N., 2010. Social cognitive conflict resolution: contributions of domain-general and domain-specific neural systems. J. Neurosci. 30, 8481-8488. doi:10.1523/JNEUROSCI.0382-10.2010. 